\title{
Gauge invariant 1PI effective superstring field theory: inclusion of the Ramond sector
}

\author{
Ashoke Sen \\ Harish-Chandra Research Institute, \\ Chhatnag Road, Jhusi, Allahabad 211019, India \\ E-mail: sen@mri.ernet.in
}

ABSTRACT: We construct off-shell amplitudes in heterotic and type II string theories involving arbitrary combination of Ramond and Neveu-Schwarz sector external states. We also construct the equations of motion of a gauge invariant 1PI effective field theory which reproduces these off-shell amplitudes. Using this construction we prove that the renormalized physical masses do not depend on the choice of local coordinate system and locations of picture changing operators used in defining the off-shell amplitudes. We also use this formalism to examine the conditions under which space-time supersymmetry is unbroken in the quantum theory.

KEYwords: Superstrings and Heterotic Strings, String Field Theory

ARXIV EPRINT: 1501.00988 


\section{Contents}

1 Introduction $\quad 1$

2 Conventions and definitions 4

$\begin{array}{llr}3 & \text { Off-shell amplitudes } & 6\end{array}$

4 The 1PI effective field theory $\quad 13$

4.1 The \{\} and [ ] products 13

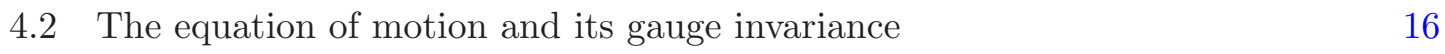

$\begin{array}{lll}4.3 & \text { Auxiliary action and S-matrix elements } & 17\end{array}$

$\begin{array}{ll}4.4 \text { Generalizaton to type II string theories } & 19\end{array}$

5 Effect of changing the local coordinates and/or PCO locations $\quad 19$

6 Space-time supersymmetry $\quad 24$

$\begin{array}{llr}7 & \text { Discussion } & 26\end{array}$

\section{Introduction}

In a previous paper [1] we constructed a gauge invariant one particle irreducible (1PI) effective action involving Neveu-Schwarz (NS) sector fields of heterotic string theories and NSNS sector fields of type II string theories. This led to a well defined algorithm for computing the renormalized masses of physical states. Furthermore it was possible to show that the renormalized physical masses are independent of the choice of any spurious data e.g. the choice of local coordinates at the punctures or the locations of the picture changing operator (PCO)'s [2] used in the construction of the 1PI action. The goal of this paper is to extend the construction to the Ramond (R) sector for heterotic string theory and RNS, NSR and RR sectors of the type II string theory.

It has been known since the early days of string field theory [3] that a straightforward construction of a gauge invariant string field theory action involving R-sector states is likely to fail due to the difficulty in the construction of the kinetic term of the R-sector string fields. The difficulty has its origin in the fact that unlike in the case of -1 picture NS sector states where the BPZ inner product between two such states has the right picture number $(-2)$ for giving a non-zero answer, the two $\mathrm{R}$ sector states in the $-1 / 2$ picture cannot have non-zero BPZ inner product unless we insert additional operators of picture number -1 into the matrix element. This makes it difficult to write down a kinetic term for the $\mathrm{R}$ sector fields that is local, commutes with $L_{0}^{ \pm}$so that it does not mix states at 
different levels and whose cohomology coincides with the usual BRST cohomology. ${ }^{1}$ There is also an indirect argument from low energy effective field theory which goes as follows. If we did manage to write down a kinetic term for the $\mathrm{R}$ sector fields in a straightforward manner then we could also use it to write down a gauge invariant kinetic term for the RR 4 -form field of type IIB string theory. But we know that it should not be possible to write down a covariant action for the RR 4-form field due to the self-duality constraint on its field strength.

We circumvent this problem by giving up the attempt to construct a gauge invariant local 1PI action involving R-sector fields. Instead we construct the gauge invariant 1PI effective equations of motion. ${ }^{2}$ If we were trying to construct a string field theory action that needs to be quantized then having equations of motion is not very useful - one needs the action for being able to quantize the theory. However the 1PI effective theory by definition already includes the effect of loop corrections and we are supposed to compute tree amplitudes of this theory to find the full quantum corrected S-matrix of string theory. Thus having the equations of motion of the 1PI effective theory is sufficient for our purpose.

We have not attempted to make the paper self-contained - it should be regarded as the completion of the program described in $[1,10]$. Nevertheless we review the main conventions in section 2. As the rest of the paper is mostly technical in nature, we shall try to summarize the main results here.

1. One of the bottlenecks faced in $[1,10]$ for generalizing the definition of off-shell amplitudes to the $\mathrm{R}$ sector is finding a suitable definition of the gluing compatibility condition. This is equivalent to the problem of finding a propagator in the $\mathrm{R}$ sector if we restrict the $\mathrm{R}$ sector string fields to carry picture number $-1 / 2$. In section 3 we make a specific proposal where we insert into the usual NS sector propagator $b_{0}^{+} b_{0}^{-}\left(L_{0}^{+}\right)^{-1} \delta_{L_{0}^{-}}$a factor of $\mathcal{X}_{0} \equiv \oint z^{-1} d z \mathcal{X}(z)$ to define the propagator of $\mathrm{R}$ sector states in heterotic string theory. Here $\mathcal{X}(z)$ is the picture changing operator and $b_{0}^{ \pm}$and $L_{0}^{ \pm}$are defined in (2.5). The advantage of using the operator $\mathcal{X}_{0}$ is that it commutes with $b_{0}^{ \pm}$and $L_{0}^{ \pm}[11,12]$ and hence can be inserted anywhere on the $\mathrm{R}$ sector propagator. For the type II string we need a similar operator $\overline{\mathcal{X}}_{0}$ involving left handed PCO and insert $\mathcal{X}_{0}, \overline{\mathcal{X}}_{0}$ and $\mathcal{X}_{0} \overline{\mathcal{X}}_{0}$ into the propagator for $N S R, R N S$ and $R R$ fields. Once the propagators in different sectors are defined one can generalize the construction of off-shell amplitudes in $[1,10]$ to the Ramond sector states in a straightforward manner. This is discussed in section 3.

2. We can use this definition of off-shell amplitudes to define 1PI amplitudes by restricting the integral over the moduli space to a restricted domain such that the '1PI Riemann surfaces' associated with this restricted domain, together with all other Riemann surfaces which can be obtained by plumbing fixture of the 1PI Riemann

\footnotetext{
${ }^{1}$ It is possible to live with this problem for open strings by working with kinetic operator which is not diagonal in the $L_{0}$ basis as in [3] but the problem reappears for closed string theory in which we are interested.

${ }^{2} \mathrm{~A}$ recent attempt to construct the equations of motion of R-sector fields in the Berkovits formulation of string field theory [4-7] can be found in $[8,9]$.
} 
surfaces in all possible ways, generate all the Riemann surfaces over which we integrate to get the full off-shell amplitude. The generating functional of the 1PI amplitudes define the 1PI effective field theory whose off-shell Green's functions in the Siegel gauge would agree with the off-shell amplitudes constructed in section 3. This construction of 1 PI effective field theory is carried out in section 4 . The general string field configuration $|\Psi\rangle$ is taken to be an element of the Hilbert space of matterghost conformal field theory with picture number -1 in the NS sector and picture number $-1 / 2$ in the $\mathrm{R}$ sector. The equations of motion are given in (4.15) and its infinite dimensional gauge invariance is described in (4.16). In these equations $\mathcal{G}$ stands respectively for the identity operator and $\mathcal{X}_{0}$ while acting on the NS and R sector states of heterotic string theory. The equations of motion and gauge transformation laws of type II string theory have the same form with $\mathcal{G}$ standing respectively for the identity operator, $\mathcal{X}_{0}, \overline{\mathcal{X}}_{0}$ and $\mathcal{X}_{0} \overline{\mathcal{X}}_{0}$ while acting on the NSNS, NSR, RNS and RR sector states.

3. Even though there is no fully satisfactory 1PI effective action for this theory, in section 4.3 we show that it is possible to write down an action (4.21) from which we can derive the equations of motion. The problem with this action is that it contains extra states that are not present in string theory. For a classical theory that needs to be quantized, the presence of these extra states would be fatal since they would propagate in the loop and completely change the loop amplitudes. However since the 1PI theory is to be used only for classical / tree level computation, we can use this action to compute the S-matrix elements of string theory by restricting the external states to a subset of states which correspond to genuine physical states in string theory.

4. The definition of [ ] used in (4.15), (4.16) depends on the choice of the local coordinate system at the punctures and the PCO locations used in defining the off-shell amplitudes. In section 5 we show that the change in the equations of motion (4.15) under these changes can be absorbed into a redefinition of the string field $|\Psi\rangle$. This is turn shows that the physical renormalized masses are independent of the choices of local coordinate systems and PCO locations, generalizing the results of $[13,14]$.

5. The gauge transformation laws (4.16) automatically include local supersymmetry transformations. In section 6 we discuss the conditions under which there is unbroken global supersymmetry. Our analysis leads to a condition similar to the one found in $[15,16]$, except that we arrive at a slightly different procedure for dealing with divergences associated with separating type degenerations compared to the one suggested in $[15,16]$.

6. We conclude in section 7 by discussing possible future applications of this approach - study of non-perturbative effects in string theory and the study of string theory in RR background field. 
Finally, as in [1], we would like to emphasize that even though we have used the PCO formalism for the construction of the off-shell amplitudes and 1PI effective theory, it may also be possible to carry out similar construction in the more geometric approach where superstring amplitudes are represented as integrals over supermoduli spaces. For on-shell amplitudes such a formalism has already been developed (see [15-33] for recent developments). It is also conceivable that once such a formalism is developed for off-shell amplitudes and 1PI effective theory, one should also be able to show its equivalence with the formalism developed here based on picture changing operators.

\section{Conventions and definitions}

We shall follow the notations of $[1,10]$. We begin our discussion with heterotic string theory. In this case the world sheet theory contains a matter superconformal field theory with central charge $(26,15)$, and a ghost system of total central charge $(-26,-15)$ containing anti-commuting $b, c, \bar{b}, \bar{c}$ ghosts and commuting $\beta, \gamma$ ghosts. Of these $b, c, \beta, \gamma$ are righthanded and $\bar{b}, \bar{c}$ are left-handed. The $(\beta, \gamma)$ system can be bosonized as [2]

$$
\gamma=\eta e^{\phi}, \quad \beta=\partial \xi e^{-\phi}, \quad \delta(\gamma)=e^{-\phi}, \quad \delta(\beta)=e^{\phi},
$$

where $\xi, \eta$ are fermions and $\phi$ is a scalar with background charge. The (ghost number, picture number, GSO) quantum numbers carried by various fields are as follows:

$$
\begin{array}{cccc}
c, \bar{c}:(1,0,+), & b, \bar{b}:(-1,0,+), & \gamma:(1,0,-), \quad \beta:(-1,0,-), \\
\xi:(-1,1,+), & \eta:(1,-1,+), & e^{q \phi}:\left(0, q,(-1)^{q}\right) . &
\end{array}
$$

We denote by $Q_{B}$ the BRST operator of this theory and by $\mathcal{X}(z)$ the picture changing operator

$$
\mathcal{X}(z)=\left\{Q_{B}, \xi(z)\right\}=c \partial \xi+e^{\phi} T_{F}-\frac{1}{4} \partial \eta e^{2 \phi} b-\frac{1}{4} \partial\left(\eta e^{2 \phi} b\right) .
$$

This is a BRST invariant dimension zero primary operator and carries picture number 1 .

We now introduce vector spaces $\mathcal{H}_{(n)}$ containing a subset of GSO even states in the matter-ghost conformal field theory satisfying the following conditions:

$$
|s\rangle \in \mathcal{H}_{(n)} \quad \text { iff } \quad b_{0}^{-}|s\rangle=0, \quad L_{0}^{-}|s\rangle=0, \quad \eta_{0}|s\rangle=0, \quad \text { picture number of }|s\rangle=n,
$$

where

$$
b_{0}^{ \pm} \equiv\left(b_{0} \pm \bar{b}_{0}\right), \quad L_{0}^{ \pm} \equiv\left(L_{0} \pm \bar{L}_{0}\right), \quad c_{0}^{ \pm}=\frac{1}{2}\left(c_{0} \pm \bar{c}_{0}\right)
$$

Note that $\mathcal{H}_{(n)}$ contains NS-sector states for $n \in \mathbb{Z}$ and R-sector states for $n \in \mathbb{Z}+\frac{1}{2}$. Although eventually we shall be interested in states for which the coefficient of the NS-sector states are even elements of the grassmann algebra and the coefficients of the R-sector states are odd elements of the grassmann algebra, for now we shall work with a more general space in which we allow the coefficients in each $\mathcal{H}_{(n)}$ to be a general element of the grassmann algebra. We shall also define

$$
\mathcal{H}_{N S}=\oplus_{n \in \mathbb{Z}} \mathcal{H}_{(n)}, \quad \mathcal{H}_{R}=\oplus_{n \in \mathbb{Z}+\frac{1}{2}} \mathcal{H}_{(n)}, \quad \mathcal{H}_{T}=\mathcal{H}_{N S} \oplus \mathcal{H}_{R}
$$


In the construction of string field theory a general off-shell string field configuration will be represented by an element of $\mathcal{H}_{T}$ with ghost number 2 and picture numbers -1 or $-1 / 2$. However for now we shall work with general states in $\mathcal{H}_{T}$.

Next we introduce the operator - introduced earlier in $[11,12,34,35]$ for construction of string field theory action in the NS sector,

$$
\mathcal{X}_{0}=\oint \frac{d z}{z} \mathcal{X}(z)
$$

where the integration runs around an anti-clockwise contour enclosing the origin with the factor of $1 / 2 \pi i$ included in its definition. We need to treat $\mathcal{X}_{0}$ as an operator in radial quantization, acting on states represented by vertex operators at the origin. The important properties of $\mathcal{X}_{0}$ are its commutation relations [11, 12]

$$
\left[b_{0}, \mathcal{X}_{0}\right]=0, \quad\left[L_{0}, \mathcal{X}_{0}\right]=0, \quad\left[\bar{b}_{0}, \mathcal{X}_{0}\right]=0, \quad\left[\bar{L}_{0}, \mathcal{X}_{0}\right]=0, \quad\left[Q_{B}, \mathcal{X}_{0}\right]=0
$$

The first identity requires some discussion. Using (2.3) we get

$$
\left[b_{0}, \mathcal{X}_{0}\right]=\oint d z \partial \xi(z)
$$

This would vanish if $\xi(z)$ is single valued. Now even though $\xi(z)$ is not an allowed conformal field in the small Hilbert space that we are working in [2] — encoded in the $\eta_{0}|s\rangle=0$ condition in (2.4) — it was shown in [36] that all the correlation functions of $\xi(z)$ on arbitrary Riemann surfaces are indeed single-valued. In terms of operators in the small Hilbert space this means that $\oint d z \partial \xi(z)$ vanishes for integration over any closed contour on the Riemann surface. This leads to the first equations in (2.8). The other equations follow in a straightforward manner.

For convenience we shall define the general operator $\mathcal{G}$ acting on $\mathcal{H}_{T}$ as

$$
\mathcal{G}|s\rangle=\left\{\begin{array}{l}
|s\rangle \quad \text { if }|s\rangle \in \mathcal{H}_{N S} \\
\mathcal{X}_{0}|s\rangle \quad \text { if }|s\rangle \in \mathcal{H}_{R}
\end{array}\right.
$$

For type II string theories we also have left-handed commuting ghosts $\bar{\beta}, \bar{\gamma}$ which can be bosonized as in (2.1), introducing the fields $\bar{\xi}, \bar{\eta}, \bar{\phi}$. We also need to introduce left-handed GSO quantum numbers and picture numbers and declare that the right-handed fields are neutral under the left-handed GSO and left-handed picture numbers while the left-handed fields are neutral under the right-handed GSO and right-handed picture numbers. However for the ghost number we do not distinguish between left and right handed sectors so that the $\bar{\xi}, \bar{\eta}, e^{q \bar{\phi}}$ carry the same ghost numbers as their right-handed counterpart. We introduce the left-handed PCO

$$
\overline{\mathcal{X}}(\bar{z})=\left\{Q_{B}, \bar{\xi}(\bar{z})\right\}=\bar{c} \bar{\partial} \bar{\xi}+e^{\bar{\phi}} \bar{T}_{F}-\frac{1}{4} \bar{\partial} \bar{\eta} e^{2 \bar{\phi}} \bar{b}-\frac{1}{4} \bar{\partial}\left(\bar{\eta} e^{2 \bar{\phi}} \bar{b}\right)
$$

and

$$
\overline{\mathcal{X}}_{0}=\oint \frac{d \bar{z}}{\bar{z}} \overline{\mathcal{X}}(\bar{z})
$$


The relevant states in the Hilbert space can now be divided into the subspaces $\mathcal{H}_{(m, n)}$ where $m$ and $n$ denote respectively the left and the right-handed picture numbers. Each sector contains states that are annihilated by $L_{0}^{-}, b_{0}^{-}, \eta_{0}$ and $\bar{\eta}_{0}$. The analog of (2.6) is

$$
\begin{array}{rlrl}
\mathcal{H}_{N S N S} & =\oplus_{m, n \in \mathbb{Z}} \mathcal{H}_{(m, n)}, & \mathcal{H}_{N S R} & =\oplus_{m \in \mathbb{Z}, n \in \mathbb{Z}+\frac{1}{2}} \mathcal{H}_{(m, n)}, \\
\mathcal{H}_{R N S} & =\oplus_{m \in \mathbb{Z}+\frac{1}{2}, n \in \mathbb{Z}} \mathcal{H}_{(m, n)}, & \mathcal{H}_{R R} & =\oplus_{m, n \in \mathbb{Z}+\frac{1}{2}} \mathcal{H}_{(m, n)}, \\
\mathcal{H}_{T} & =\mathcal{H}_{N S N S} \oplus \mathcal{H}_{N S R} \oplus \mathcal{H}_{R N S} \oplus \mathcal{H}_{R R} . &
\end{array}
$$

Finally we define

$$
\mathcal{G}|s\rangle=\left\{\begin{array}{l}
|s\rangle \quad \text { if }|s\rangle \in \mathcal{H}_{N S N S} \\
\mathcal{X}_{0}|s\rangle \quad \text { if }|s\rangle \in \mathcal{H}_{N S R} \\
\overline{\mathcal{X}}_{0}|s\rangle \quad \text { if }|s\rangle \in \mathcal{H}_{R N S} \\
\mathcal{X}_{0} \overline{\mathcal{X}}_{0}|s\rangle \quad \text { if }|s\rangle \in \mathcal{H}_{R R}
\end{array}\right.
$$

\section{Off-shell amplitudes}

$g$-loop, $n$-point on-shell amplitude in bosonic string theory is expressed as an integral over the $(6 g-6+2 n)$ dimensional moduli space $\mathcal{M}_{g, n}$ of genus $g$ Riemann surfaces with $n$-punctures. Defining off-shell amplitudes in bosonic string theory requires extra data in the form of a choice of local coordinate system around each puncture. This requires us to introduce an infinite dimensional space $\widehat{\mathcal{P}}_{g, n}$ with the structure of a fiber bundle whose base is $\mathcal{M}_{g, n}$ and whose (infinite dimensional) fiber is parametrized by the possible choices of local coordinate system around each puncture [37, 38]. The off-shell amplitude is described as an integral of a $(6 g-6+2 n)$-form over a section of $\widehat{\mathcal{P}}_{g, n}$. The construction of the differential form to be integrated as well as the subspaces over which we need to integrate can be found in [10] (and also reviewed in [1]).

Defining off-shell amplitude in heterotic and type II string theories requires even more data - a choice of the locations of certain number of PCO's on the Riemann surface. Let us for definiteness focus on the heterotic string theory - generalization to type II string theories will be discussed later. A genus $g$ amplitude in heterotic string theory with $m$ NS sector external states in the -1 picture and $n$ Ramond sector external states in the $-1 / 2$ picture requires a total of $2 g-2+m+n / 2$ PCO insertions. Even though we shall need to relax the constraint on the picture number on the states for various manipulations, the off-shell amplitudes that we shall need will always involve NS-sector external states in the -1 picture and R-sector external states in the $-1 / 2$ picture, and hence we shall always use the same number of PCO insertions on a genus $g$ Riemann surface with $m$ NS-sector external states and $n$ R-sector external states. Thus we need to introduce a bigger fiber-bundle $\widetilde{\mathcal{P}}_{g, m, n}$ with $\mathcal{M}_{g, m, n}$ - the moduli space of genus $g$ Riemann surface with $m$ NS-punctures and $n$ R-punctures - as base and the choice of local coordinates at the punctures and the $2 g-2+m+n / 2$ PCO locations as fibers. The off-shell amplitude is defined as an integral of an appropriate differential form of degree $6 g-6+2(m+n)$ over an appropriate $6 g-2+2(m+n)$-dimensional subspace 
of this fiber bundle (together with a sum over spin structures which we shall include in the definition of the integral). In fact, following the procedure described in [10] we can explicitly construct a set of $p$ forms $\Omega_{p}^{(g, m, n)}\left(\left|\tilde{\phi}_{1}\right\rangle, \cdots\left|\tilde{\phi}_{m}\right\rangle,\left|\hat{\phi}_{1}\right\rangle, \cdots\left|\hat{\phi}_{n}\right\rangle\right)$ on $\mathcal{P}_{g, m, n}$ for all $p$, satisfying useful identities to be discussed in (3.4), (3.6), (3.8), (3.10), whose the first $m$ arguments $\left|\tilde{\phi}_{1}\right\rangle, \cdots\left|\tilde{\phi}_{m}\right\rangle$ are NS sector states and the last $n$ arguments $\left|\hat{\phi}_{1}\right\rangle, \cdots\left|\hat{\phi}_{n}\right\rangle$ are R-sector states. $\Omega_{p}^{(g, m, n)}\left(\left|\tilde{\phi}_{1}\right\rangle, \cdots\left|\tilde{\phi}_{m}\right\rangle,\left|\hat{\phi}_{1}\right\rangle, \cdots\left|\hat{\phi}_{n}\right\rangle\right)$ is expressed in terms of a correlation function in the matter-ghost CFT with the state $\left|\tilde{\phi}_{i}\right\rangle \in \mathcal{H}_{N S}$ and $\left|\hat{\phi}_{j}\right\rangle \in \mathcal{H}_{R}$ inserted at the $i$-th and $(m+j)$-th punctures together with $p$ additional insertion of $b$ or $\bar{b}$ ghost fields and $2 g-2+m+n / 2 \mathrm{PCO}$ insertions. Ghost and picture number conservations tell us that $\Omega_{p}^{(g, m, n)}$ is non-zero only if the total ghost number of $\left|\tilde{\phi}_{1}\right\rangle, \cdots\left|\tilde{\phi}_{m}\right\rangle,\left|\hat{\phi}_{1}\right\rangle, \cdots\left|\hat{\phi}_{n}\right\rangle$ is $p-6 g+6$ and the total picture number of $\left|\tilde{\phi}_{1}\right\rangle, \cdots\left|\tilde{\phi}_{m}\right\rangle,\left|\hat{\phi}_{1}\right\rangle, \cdots\left|\hat{\phi}_{n}\right\rangle$ is $-m-\frac{1}{2} n .^{3}$

Compared to the case of bosonic string theory, there are some new subtleties that arise in the choice of the subspace of $\widetilde{\mathcal{P}}_{g, m, n}$ over which we integrate. These are listed below:

1. As discussed in [10], generically the subspace of $\widetilde{\mathcal{P}}_{g, m, n}$ over which we need to integrate contains vertical segments - along which the location of the PCO's change at fixed values of the coordinates of the base $\mathcal{M}_{g, m, n}$ - in order to avoid spurious singularities $[36,39,40]$. The procedure for carrying out integrals over these vertical segments was described in [10] and works equally well for NS or R sector external states. Since subspaces containing vertical segments are not strictly sections of $\widetilde{\mathcal{P}}_{g, m, n}$, we refer to these as integration cycles.

2. It may not always be possible to have a fixed subspace of $\widetilde{\mathcal{P}}_{g, m, n}$ that is consistent with all the symmetries e.g. modular invariance and symmetry under the permutations of external punctures. ${ }^{4}$ In order to deal with this problem we allow the integration cycle to be formal weighted average of several subspaces. The integral of a form on a formal weighted average of several subspaces is defined as the weighted average of the integrals of the form over different subspaces. From now on, when we refer to subspaces of $\widetilde{\mathcal{P}}_{g, m, n}$, they will in general mean weighted average of subspaces.

3. The third subtlety arises while dealing with off-shell amplitudes with Ramond sector external states. The problem has its origin in the fact that in order to ensure that the off-shell amplitude leads to sensible definition of physical quantities - e.g. renormalized physical masses and S-matrix elements - we need to ensure that the choice of the integration cycle is gluing compatible. To see what it means, recall that if we consider two Riemann surfaces $\Sigma_{1}$ and $\Sigma_{2}$, and pick one puncture on each of them with local coordinates $z$ and $w$, then we can construct a two parameter family of Riemann surfaces $\Sigma$ by joining $\Sigma_{1}$ and $\Sigma_{2}$ using the plumbing fixture relation:

$$
z w=e^{-s+i \theta}, \quad 0 \leq s<\infty, \quad 0 \leq \theta<2 \pi .
$$

\footnotetext{
${ }^{3}$ Even though the emphasis in [10] was on the NS sector external states for reasons to be explained below, the construction of $\Omega_{p}^{(g, m, n)}\left(\left|\tilde{\phi}_{1}\right\rangle, \cdots\left|\tilde{\phi}_{m}\right\rangle,\left|\hat{\phi}_{1}\right\rangle, \cdots\left|\hat{\phi}_{n}\right\rangle\right)$ itself can be carried out in an identical manner irrespective of whether the external states are NS or R-sector states.

${ }^{4}$ Here we shall only demand symmetry under the permutation of the NS sector punctures and separately under the permutation of R-sector punctures.
} 
Gluing compatibility requires that on the resulting Riemann surfaces $\Sigma$ the choice of local coordinates as well as the locations of the PCO's will be induced by those from the original Riemann surfaces $\Sigma_{1}$ and $\Sigma_{2}$. This does not cause any problem when the two punctures which are glued are associated with NS-sector states. To see this we note that if $\Sigma_{1}$ has genus $g_{1}$ with $n_{N 1}$ NS punctures and $n_{R 1} \mathrm{R}$ punctures and $\Sigma_{2}$ has genus $g_{2}$ with $n_{N 2}$ NS punctures and $n_{R 2} \mathrm{R}$ punctures, then we have $2 g_{1}-2+n_{N 1}+n_{R 1 / 2}$ PCO's on $\Sigma_{1}$ and $2 g_{2}-2+n_{N 2}+n_{R 2 / 2}$ PCO's on $\Sigma_{2}$. The sum of these matches the required number of PCO's on $\Sigma$ which has genus $g_{1}+g_{2}$, $\left(n_{N_{1}}+n_{N 2}-2\right)$ NS-punctures and $\left(n_{R 1}+n_{R 2}\right)$ R-punctures. However if the two punctures being glued are of R-type, then $\Sigma$ has genus $g_{1}+g_{2},\left(n_{N_{1}}+n_{N 2}\right)$ NSpunctures and $\left(n_{R 1}+n_{R 2}-2\right)$ R-punctures. The required number of PCO's on $\Sigma$ is $2\left(g_{1}+g_{2}\right)+2\left(n_{N 1}+n_{N 2}\right)+\left(n_{R 1}+n_{R 2}\right) / 2-3$ which is one more than the total number of PCO's on $\Sigma_{1}$ and $\Sigma_{2}$. For this reason the analysis in [10] was restricted mostly to NS sector external states.

In this paper we propose a prescription for the choice of the PCO's on $\Sigma$ when the punctures being glued are Ramond punctures. Our prescription will be to choose $2\left(g_{1}+\right.$ $\left.g_{2}\right)+2\left(n_{N 1}+n_{N 2}\right)+\left(n_{R 1}+n_{R 2}\right) / 2-4$ of the PCO locations to be those induced from $\Sigma_{1}$ and $\Sigma_{2}$ and the last PCO to be $\mathcal{X}_{0}$ given in (2.7). In other words we do not insert the extra $\mathrm{PCO}$ at a single point but take a formal weighted average of infinite number of insertions given by

$$
\mathcal{X}_{0} \equiv \oint \frac{d z}{z} \mathcal{X}(z)
$$

The contour of integration can be taken to be any anti-clockwise contour with $e^{-s} \leq|z| \leq 1$. This translates to the same condition on $w=e^{-s+i \theta} / z$. Furthermore even though $d z / z=$ $-d w / w$, an anti-clockwise contour in the $z$-plane corresponds to a clockwise contour in the $w$ plane. Thus the prescription is symmetric between the two punctures.

Once a gluing compatible integration cycle has been chosen this way, we can define the off-shell amplitude for $m$ NS-sector external states $\left|\tilde{\phi}_{1}\right\rangle, \cdots\left|\tilde{\phi}_{m}\right\rangle$ and $n$ R-sector external states $\left|\hat{\phi}_{1}\right\rangle, \cdots\left|\hat{\phi}_{n}\right\rangle$ by integrating $\Omega_{6 g-6+2(m+n)}^{(g, m, n)}\left(\left|\tilde{\phi}_{1}\right\rangle, \cdots\left|\tilde{\phi}_{m}\right\rangle,\left|\hat{\phi}_{1}\right\rangle, \cdots\left|\hat{\phi}_{n}\right\rangle\right)$ over this integration cycle. Of course in order to prove the usefulness of this prescription we need to show that the physical quantities computed from this prescription, e.g. the renormalized masses and S-matrix elements, are independent of the choice of the integration cycles used in defining the off-shell amplitude. This will be done in the next sections by turning this into a prescription for constructing a gauge invariant effective field theory and then showing that the effect of changing the integration cycles can be absorbed into a field redefinition.

We now address a few issues associated with this prescription:

1. Suppose we have chosen the locations of the PCO's on $\Sigma_{1}$ and $\Sigma_{2}$ so as to avoid spurious poles. Is it guaranteed that the relevant correlation function on $\Sigma$, with the PCO arrangements as described above, is free from spurious singularities? As described in [10], if we choose the local coordinates $z$ and $w$ at the punctures being glued in such a way that $|z| \leq 1$ and $|w| \leq 1$ describe sufficiently small disks around 
the respective punctures, then the relevant correlation function on $\Sigma$ is given approximately by the sum of products of correlation functions on $\Sigma_{1}$ and $\Sigma_{2}$ with states of low $L_{0}^{+}$inserted at the punctures that are being glued and the matrix element of $b_{0}^{+} b_{0}^{-}\left(L_{0}^{+}\right)^{-1}$ (or $b_{0}^{+} b_{0}^{-}\left(L_{0}^{+}\right)^{-1} \mathcal{X}_{0}$ ) between these low $L_{0}^{+}$states if they belong to the NS sector (or R-sector). This is free from spurious singularity by construction. We shall always choose the local coordinate systems at the punctures in this manner.

2. The family of Riemann surfaces described in (3.1) has a boundary at $s=0$. This is not a boundary of the moduli space and hence the full integration cycle must involve Riemann surfaces which lie beyond this boundary. On these Riemann surfaces the choice of PCO's is not restricted by the choice of locations of the PCO's on $\Sigma_{1}$ and $\Sigma_{2}$ except that we require the choice of PCO locations to be continuous across this boundary. If we require the choice of PCO locations to be continuous everywhere in the moduli space then we'll need to continue using the averages over PCO locations like the one given in (3.2) everywhere in the moduli space. However this is not necessary, since using the rules for 'integration across vertical segments' we can allow the PCO locations to jump discontinuously across codimension one subspaces of the moduli space. We now give an example of such a construction. With the choice of local coordinates of the type described above we expect that we can continue to choose the PCO insertions of the type we have used till $s=-\epsilon$ for sufficiently small $\epsilon$ without encountering any spurious pole. Now we can choose the PCO locations such that over the codimension one subspace of the moduli space given by $s=-\epsilon$ all the PCO locations labelled by $z$ in (3.2) change to some fixed value $z_{0}$. According to the prescription given in [10] this will require integrating over this subspace of the moduli space an appropriate differential form whose construction involves the insertion of (see e.g. eq. (3.45) of [10])

$$
\oint \frac{d z}{z}\left(\xi(z)-\xi\left(z_{0}\right)\right)
$$

into the correlation function. In this case beyond the $s=-\epsilon$ subspace we can use the extra PCO location to be at some fixed point $z_{0}$ instead of being distributed over a circle. More generally the integration cycle can contain different segments in which the character of the PCO locations could change, with some segments containing all the PCO locations at fixed points on the Riemann surface, while the other segments having one or more of the PCO locations averaged over insertions over one (or even two) dimensional subspaces of the Riemann surface.

We can now proceed in a manner identical to that in $[1,10]$ and introduce the $6 g-6+$ $2(m+n)$ dimensional subspaces $\mathcal{R}_{g, m, n}$ of $\widetilde{\mathcal{P}}_{g, m, n}$, known as 1 PI subspaces, such that by gluing the Riemann surfaces associated with $\mathcal{R}_{g, m, n}$ in all possible ways using the plumbing fixture relation (3.1) we generate all Riemann surfaces associated with the full integration cycle used to define the off-shell amplitudes. We list below the important properties of $\Omega_{p}^{(g, m, n)}$ and $\mathcal{R}_{g, m, n}$ 
We begin with the properties of $\Omega_{p}^{(g, m, n)}$. First of all, we have

$$
\begin{aligned}
& \sum_{i=1}^{m}(-1)^{\tilde{\gamma}_{1}+\cdots \tilde{\gamma}_{i-1}} \Omega_{p}^{(g, m, n)}\left(\left|\tilde{\phi}_{1}\right\rangle, \cdots\left|\tilde{\phi}_{i-1}\right\rangle, Q_{B}\left|\tilde{\phi}_{i}\right\rangle,\left|\tilde{\phi}_{i+1}\right\rangle, \cdots\left|\tilde{\phi}_{m}\right\rangle,\left|\hat{\phi}_{1}\right\rangle, \cdots\left|\hat{\phi}_{n}\right\rangle\right) \\
& +\sum_{i=1}^{n}(-1)^{\tilde{\gamma}_{1}+\cdots \tilde{\gamma}_{m}+\hat{\gamma}_{1}+\cdots \hat{\gamma}_{i-1}} \Omega_{p}^{(g, m, n)}\left(\left|\tilde{\phi}_{1}\right\rangle, \cdots\left|\tilde{\phi}_{m}\right\rangle,\left|\hat{\phi}_{1}\right\rangle, \cdots\left|\hat{\phi}_{i-1}\right\rangle, Q_{B}\left|\hat{\phi}_{i}\right\rangle,\left|\hat{\phi}_{i+1}\right\rangle, \cdots\left|\hat{\phi}_{n}\right\rangle\right) \\
= & (-1)^{p} d \Omega_{p-1}^{(g, m, n)}\left(\left|\tilde{\phi}_{1}\right\rangle, \cdots,\left|\tilde{\phi}_{m}\right\rangle,\left|\hat{\phi}_{1}\right\rangle, \cdots\left|\hat{\phi}_{n}\right\rangle\right),
\end{aligned}
$$

where $d$ denotes exterior derivative on $\widetilde{\mathcal{P}}_{g, m, n}$ and

$$
\tilde{\gamma}_{i}=\text { grassmannality of }\left|\tilde{\phi}_{i}\right\rangle, \quad \hat{\gamma}_{i}=\text { grassmannality of }\left|\hat{\phi}_{i}\right\rangle,
$$

the grassmannality of an operator being defined as 0 (1) if the operator is grassmann even (odd). The grassmannality of a GSO even operator in the matter ghost conformal field theory is equal to its ghost number mod 2 in the NS sector and ghost number +1 mod 2 in the $\mathrm{R}$ sector if the coefficient multiplying the operator is grassmann even. If the coefficient is grassmann odd then the grassmannality will be opposite. In the same convention, $\Omega_{p}^{(g, m, n)}$ has the symmetry property

$$
\begin{aligned}
& s_{i, i+1} \circ \Omega_{p}^{(g, m, n)}\left(\left|\tilde{\phi}_{1}\right\rangle, \cdots\left|\tilde{\phi}_{i-1}\right\rangle,\left|\tilde{\phi}_{i+1}\right\rangle,\left|\tilde{\phi}_{i}\right\rangle,\left|\tilde{\phi}_{i+2}\right\rangle \cdots\left|\tilde{\phi}_{m}\right\rangle,\left|\hat{\phi}_{1}\right\rangle, \cdots\left|\hat{\phi}_{n}\right\rangle\right) \\
= & (-1)^{\tilde{\gamma}_{i} \tilde{\gamma}_{i+1}} \Omega_{p}^{(g, m, n)}\left(\left|\tilde{\phi}_{1}\right\rangle, \cdots\left|\tilde{\phi}_{m}\right\rangle,\left|\hat{\phi}_{1}\right\rangle, \cdots\left|\hat{\phi}_{n}\right\rangle\right) \\
& s_{m+i, m+i+1} \circ \Omega_{p}^{(g, m, n)}\left(\left|\tilde{\phi}_{1}\right\rangle, \cdots\left|\tilde{\phi}_{m}\right\rangle,\left|\hat{\phi}_{1}\right\rangle, \cdots\left|\hat{\phi}_{i-1}\right\rangle,\left|\hat{\phi}_{i+1}\right\rangle,\left|\hat{\phi}_{i}\right\rangle,\left|\hat{\phi}_{i+2}\right\rangle \cdots\left|\hat{\phi}_{n}\right\rangle\right) \\
= & (-1)^{\hat{\gamma}_{i} \hat{\gamma}_{i+1}} \Omega_{p}^{(g, m, n)}\left(\left|\tilde{\phi}_{1}\right\rangle, \cdots\left|\tilde{\phi}_{m}\right\rangle,\left|\hat{\phi}_{1}\right\rangle, \cdots\left|\hat{\phi}_{n}\right\rangle\right),
\end{aligned}
$$

where $s_{i, i+1}$ is the transformation on $\widetilde{\mathcal{P}}_{g, m, n}$ that exchanges the punctures $i$ and $i+1$ together with their local coordinates and $s_{i, i+1} \circ \Omega_{p}^{(g, m, n)}$ is the pullback of $\Omega_{p}^{(g, m, n)}$ under this transformation.

Let us now turn to the properties of $\mathcal{R}_{g, m, n}$. First of all, $\mathcal{R}_{g, m, n}$ is taken to be symmetric under the exchange of any pair of NS-punctures and also under the exchange of any pair of R-punctures. This needs to be achieved, if necessary, by taking $\mathcal{R}_{g, m, n}$ to be formal weighted average of subspaces related by these exchange transformations. Plumbing fixture of $\mathcal{R}_{g_{1}, m_{1}, n_{1}}$ and $\mathcal{R}_{g_{2}, m_{2}, n_{2}}$ at an NS puncture produces a subspace of $\widetilde{\mathcal{P}}_{g_{1}+g_{2}, m_{1}+m_{2}-2, n_{1}+n_{2}}$ which we shall denote by $\mathcal{R}_{g_{1}, m_{1}, n_{1}} \circ \mathcal{R}_{g_{2}, m_{2}, n_{2}}$. On the other hand plumbing fixture of $\mathcal{R}_{g_{1}, m_{1}, n_{1}}$ and $\mathcal{R}_{g_{2}, m_{2}, n_{2}}$ at an $\mathrm{R}$ puncture produces a subspace of $\widetilde{\mathcal{P}}_{g_{1}+g_{2}, m_{1}+m_{2}, n_{1}+n_{2}-2}$ which we shall denote by $\mathcal{R}_{g_{1}, m_{1}, n_{1}} \star \mathcal{R}_{g_{2}, m_{2}, n_{2}}$. Note that the insertion of the extra PCO (3.2) is included in the definition of $\mathcal{R}_{g_{1}, m_{1}, n_{1}} \star \mathcal{R}_{g_{2}, m_{2}, n_{2}}$. For definiteness let us choose the convention that the plumbing fixture will always be done with the last (NS or R) puncture of the first Riemann surface and the first (NS or R) puncture of the second Riemann surface. Furthermore on the Riemann surfaces associated with $\mathcal{R}_{g_{1}, m_{1}, n_{1}} \circ \mathcal{R}_{g_{2}, m_{2}, n_{2}}$ the first set of $m_{1}-1$ NS-punctures and $n_{1}$ R-punctures will represent the punctures on the surfaces corresponding to $\mathcal{R}_{g_{1}, m_{1}, n_{1}}$ and the last set of $m_{2}-1$ NS-punctures and $n_{2}$ R-punctures will represent the punctures on the surfaces corresponding to $\mathcal{R}_{g_{2}, m_{2}, n_{2}}$. A similar convention will be followed for the punctures on the surfaces associated with $\mathcal{R}_{g_{1}, m_{1}, n_{1}} \star \mathcal{R}_{g_{2}, m_{2}, n_{2}}$. 
The subspaces $\mathcal{R}_{g_{1}, m_{1}, n_{1}} \circ \mathcal{R}_{g_{2}, m_{2}, n_{2}}$ and $\mathcal{R}_{g_{1}, m_{1}, n_{1}} \star \mathcal{R}_{g_{2}, m_{2}, n_{2}}$ have natural boundaries containing the Riemann surfaces obtained by setting $s=0$ in the plumbing fixture relations (3.1). We shall denote them by $\left\{\mathcal{R}_{g_{1}, m_{1}, n_{1}}, \mathcal{R}_{g_{2}, m_{2}, n_{2}}\right\}$ and $\left\{\mathcal{R}_{g_{1}, m_{1}, n_{1}} ; \mathcal{R}_{g_{2}, m_{2}, n_{2}}\right\}$ respectively. Thus $\left\{\mathcal{R}_{g_{1}, m_{1}, n_{1}}, \mathcal{R}_{g_{2}, m_{2}, n_{2}}\right\}$ represents the set of punctured Riemann surfaces equipped with choice of local coordinates at the punctures and PCO locations that we obtain by gluing the families of Riemann surfaces corresponding to $\mathcal{R}_{g_{1} . m_{1}, n_{1}}$ and $\mathcal{R}_{g_{2}, m_{2}, n_{2}}$ at NS punctures using plumbing fixture relation (3.1) with the parameter $s$ set to zero. $\left\{\mathcal{R}_{g_{1}, m_{1}, n_{1}} ; \mathcal{R}_{g_{2}, m_{2}, n_{2}}\right\}$ has a similar interpretation except that the plumbing fixture is done at Ramond punctures, and we insert an extra PCO given by (3.2) around the punctures. The orientations of $\{A, B\}$ and $\{A ; B\}$ will be defined by taking its volume form to be $d \theta \wedge d V_{A} \wedge d V_{B}$ where $d V_{A}$ and $d V_{B}$ are volume forms on $A$ and $B$ respectively.

The boundaries of $\mathcal{R}_{g, m, n}$ are of special interest. Since $\mathcal{M}_{g, m, n}$ has boundaries corresponding to separating and non-separating type degenerations, the fibers over these boundaries correspond to boundaries of $\widetilde{\mathcal{P}}_{g, m, n}$. If $\mathcal{R}_{g, m, n}$ intersects these boundaries of $\widetilde{\mathcal{P}}_{g, m, n}$ then these will form boundaries of $\mathcal{R}_{g, m, n}$. But by construction $\mathcal{R}_{g, m, n}$ does not intersect the boundaries of $\widetilde{\mathcal{P}}_{g, m, n}$ corresponding to separating type degenerations - they all arise from the $s \rightarrow \infty$ limit of the plumbing fixture of two or more 1PI Riemann surfaces and hence lie in the $1 \mathrm{PR}$ region of the full integration cycle. On the other hand although $\mathcal{R}_{g, m, n}$ does intersect the boundaries of $\widetilde{\mathcal{P}}_{g, m, n}$ corresponding to non-separating type degenerations, we shall ignore them since integrals of total derivatives do not receive any boundary contribution from there [41, 42]. The other boundaries of $\mathcal{R}_{g, m, n}$ lie in the interior of $\widetilde{\mathcal{P}}_{g, m, n}$ and match the $s=0$ boundary of the subspaces $\mathcal{R}_{g_{1}, m_{1}, n_{1}} \circ \mathcal{R}_{g_{2}, m_{2}, n_{2}}$ or $\mathcal{R}_{g_{1}, m_{1}, n_{1}} \star \mathcal{R}_{g_{2}, m_{2}, n_{2}}$ for appropriate choices of $g_{i}, m_{i}, n_{i}$. This gives

$$
\begin{aligned}
\partial \mathcal{R}_{g, m, n}= & -\frac{1}{2} \sum_{\substack{g_{1}, g_{2} \\
g_{1}+g_{2}=g}} \sum_{\substack{m_{1}, m_{2} \\
m_{1}+m_{2}=m+2}} \sum_{\substack{n_{1}, n_{2} \\
n_{1}+n_{2}=n}} \mathbf{S}\left[\left\{\mathcal{R}_{g_{1}, m_{1}, n_{1}}, \mathcal{R}_{g_{2}, m_{2}, n_{2}}\right\}\right] \\
& -\frac{1}{2} \sum_{\substack{g_{1}, g_{2} \\
g_{1}+g_{2}=g}} \sum_{\substack{m_{1}, m_{2} \\
m_{1}+m_{2}=m}} \sum_{\substack{n_{1}, n_{2} \\
n_{1}+n_{2}=n+2}} \mathbf{S}\left[\left\{\mathcal{R}_{g_{1}, m_{1}, n_{1}} ; \mathcal{R}_{g_{2}, m_{2}, n_{2}}\right\}\right]
\end{aligned}
$$

where $\mathbf{S}$ denotes the operation of summing over inequivalent permutations of external NSsector punctures and also external R-sector punctures. Thus for example $\mathbf{S}\left[\mathcal{R}_{g_{1}, m_{1}, n_{1}} \circ\right.$ $\left.\mathcal{R}_{g_{2}, m_{2}, n_{2}}\right]$ involves sum over $\left(\begin{array}{c}m_{1}+m_{2}-2 \\ m_{1}-1\end{array}\right)$ inequivalent permutation of the external NSsector punctures and $\left(\begin{array}{c}n_{1}+n_{2} \\ n_{1}\end{array}\right)$ inequivalent permutation of the external R-sector punctures. The minus sign on the right hand side reflects that $\mathcal{R}_{g, m, n}, \mathcal{R}_{g_{1}, m_{1}, n_{1}} \circ \mathcal{R}_{g_{2}, m_{2}, n_{2}}$ and $\mathcal{R}_{g_{1}, m_{1}, n_{1}} \star \mathcal{R}_{g_{2}, m_{2}, n_{2}}$ will all have to fit together so they they form a subspace of the full integration cycle used for defining the off-shell amplitude. Thus the boundary of $\mathcal{R}_{g, m, n}$ will be oppositely oriented to those of $\mathcal{R}_{g_{1}, m_{1}, n_{1}} \circ \mathcal{R}_{g_{2}, m_{2}, n_{2}}$ and $\mathcal{R}_{g_{1}, m_{1}, n_{1}} \star \mathcal{R}_{g_{2}, m_{2}, n_{2}}$. The factors of $1 / 2$ account for the double counting due to the symmetry that exchanges the two Riemann surfaces corresponding to $\mathcal{R}_{g_{1}, m_{1}, n_{1}}$ and $\mathcal{R}_{g_{2}, m_{2}, n_{2}}$. 
Following analysis similar to that in $[1,10]$ one can show that on $\left\{\mathcal{R}_{g_{1}, m_{1}, n_{1}}, \mathcal{R}_{g_{2}, m_{2}, n_{2}}\right\}$, $\Omega_{p}^{\left(g_{1}+g_{2}, m_{1}+m_{2}-2, n_{1}+n_{2}\right)}$ satisfies the factorization property

$$
\begin{aligned}
& \int_{\theta} \Omega_{p}^{\left(g_{1}+g_{2}, m_{1}+m_{2}-2, n_{1}+n_{2}\right)}\left(\left|\tilde{\phi}_{1}\right\rangle, \cdots\left|\tilde{\phi}_{m_{1}+m_{2}-2}\right\rangle,\left|\hat{\phi}_{1}\right\rangle, \cdots\left|\hat{\phi}_{n_{1}+n_{2}}\right\rangle\right) \\
= & \sum_{\substack{p_{1}, p_{2} \\
p_{1}+p_{2}=p-1}} \tilde{\sigma}_{1} \tilde{\sigma}_{2} \tilde{\sigma}_{3} \tilde{\sigma}_{4} \tilde{\sigma}_{5} \Omega_{p_{1}}^{\left(g_{1}, m_{1}, n_{1}\right)}\left(\left|\tilde{\phi}_{1}\right\rangle, \cdots\left|\tilde{\phi}_{m_{1}-1}\right\rangle,\left|\tilde{\varphi}_{r}\right\rangle,\left|\hat{\phi}_{1}\right\rangle \cdots\left|\hat{\phi}_{n_{1}}\right\rangle\right) \\
& \wedge \Omega_{p_{2}}^{\left(g_{2}, m_{2}, n_{2}\right)}\left(\left|\tilde{\varphi}^{r}\right\rangle,\left|\tilde{\phi}_{m_{1}}\right\rangle, \cdots\left|\tilde{\phi}_{m_{1}+m_{2}-2}\right\rangle,\left|\hat{\phi}_{n_{1}+1}\right\rangle, \cdots\left|\hat{\phi}_{n_{1}+n_{2}}\right\rangle\right)
\end{aligned}
$$

where $\int_{\theta}$ denotes integration over the angular coordinate $\theta$ appearing in the plumbing fixture relation (3.1) and $\left\{\left|\tilde{\varphi}_{r}\right\rangle\right\}$ and $\left\{\left|\tilde{\varphi}^{r}\right\rangle\right\}$ are a set of dual basis of $\mathcal{H}_{N S}$ satisfying

$$
\left\langle\tilde{\varphi}^{r}\left|c_{0}^{-}\right| \tilde{\varphi}_{s}\right\rangle=\delta^{r}{ }_{s} \quad \Leftrightarrow \quad\left\langle\tilde{\varphi}_{s}\left|c_{0}^{-}\right| \tilde{\varphi}^{r}\right\rangle=\delta^{r}{ }_{s} .
$$

$\tilde{\sigma}_{1}$ is a sign that arises in changing the ordering of the vertex operators for $\left|\tilde{\phi}_{m_{1}}\right\rangle, \cdots\left|\tilde{\phi}_{m_{1}+m_{2}-2}\right\rangle,\left|\hat{\phi}_{1}\right\rangle, \cdots\left|\hat{\phi}_{n_{1}}\right\rangle$ to $\left|\hat{\phi}_{1}\right\rangle, \cdots\left|\hat{\phi}_{n_{1}}\right\rangle,\left|\tilde{\phi}_{m_{1}}\right\rangle, \cdots\left|\tilde{\phi}_{m_{1}+m_{2}-2}\right\rangle . \tilde{\sigma}_{2}$ is a sign factor that arises in moving the vertex operator for $\left|\tilde{\varphi}_{r}\right\rangle$ through those of $\left|\hat{\phi}_{1}\right\rangle, \cdots\left|\hat{\phi}_{n_{1}}\right\rangle$. The rest of the sign factors $\tilde{\sigma}_{3}, \tilde{\sigma}_{4}$ and $\tilde{\sigma}_{5}$ given in $(3.8)$ were already present in $[1,10]$ and originate from three sources. $\tilde{\sigma}_{3}$ arises because we need to move $p_{2}$ of the $b$-ghost insertions associated with $\Omega_{p_{2}}^{\left(g_{2}, m_{2}, n_{2}\right)}$ through the vertex operators of $\left|\tilde{\phi}_{1}\right\rangle, \cdots\left|\tilde{\phi}_{m_{1}-1}\right\rangle,\left|\hat{\phi}_{1}\right\rangle \cdots\left|\hat{\phi}_{n_{1}}\right\rangle$. $\tilde{\sigma}_{4}$ arises from the need to move the vertex operator of $\left|\tilde{\varphi}^{r}\right\rangle$ through the $p_{2}$ insertions of $b$ ghosts associated with $\Omega_{p_{2}}^{\left(g_{2}, m_{2}, n_{2}\right)}$. Finally $\tilde{\sigma}_{5}$ arises due to the need to move a factor of $b_{0}^{-}$ through the $p_{1}$ insertions of $b$-ghost operators associated with $\Omega_{p_{1}}^{\left(g_{1}, m_{1}, n_{1}\right)}$ and the vertex operators of $\left|\tilde{\phi}_{1}\right\rangle, \cdots\left|\tilde{\phi}_{m_{1}-1}\right\rangle,\left|\hat{\phi}_{1}\right\rangle \cdots\left|\hat{\phi}_{n_{1}}\right\rangle$.

On the other hand on $\left\{\mathcal{R}_{g_{1}, m_{1}, n_{1}} ; \mathcal{R}_{g_{2}, m_{2}, n_{2}}\right\}, \Omega_{p}^{\left(g_{1}+g_{2}, m_{1}+m_{2}, n_{1}+n_{2}-2\right)}$ satisfies the factorization property

$$
\begin{aligned}
& \int_{\theta} \Omega_{p}^{\left(g_{1}+g_{2}, m_{1}+m_{2}, n_{1}+n_{2}-2\right)}\left(\left|\tilde{\phi}_{1}\right\rangle, \cdots\left|\tilde{\phi}_{m_{1}+m_{2}}\right\rangle,\left|\hat{\phi}_{1}\right\rangle, \cdots\left|\hat{\phi}_{n_{1}+n_{2}-2}\right\rangle\right) \\
= & \sum_{\substack{p_{1}, p_{2} \\
p_{1}+p_{2}=p-1}} \hat{\sigma}_{1} \hat{\sigma}_{2} \hat{\sigma}_{3} \hat{\sigma}_{4} \hat{\sigma}_{5} \Omega_{p_{1}}^{\left(g_{1}, m_{1}, n_{1}\right)}\left(\left|\tilde{\phi}_{1}\right\rangle, \cdots\left|\tilde{\phi}_{m_{1}}\right\rangle,\left|\hat{\phi}_{1}\right\rangle, \cdots\left|\hat{\phi}_{n_{1}-1}\right\rangle,\left|\hat{\varphi}_{r}\right\rangle\right) \\
& \wedge \Omega_{p_{2}}^{\left(g_{2}, m_{2}, n_{2}\right)}\left(\left|\tilde{\phi}_{m_{1}+1}\right\rangle, \cdots\left|\tilde{\phi}_{m_{1}+m_{2}}\right\rangle, \mathcal{X}_{0}\left|\hat{\varphi}^{r}\right\rangle,\left|\hat{\phi}_{n_{1}}\right\rangle, \cdots\left|\hat{\phi}_{n_{1}+n_{2}-2}\right\rangle\right)
\end{aligned}
$$

where $\left\{\left|\hat{\varphi}_{r}\right\rangle\right\}$ and $\left\{\left|\hat{\varphi}^{r}\right\rangle\right\}$ are a set of dual basis of $\mathcal{H}_{R}$ satisfying

$$
\left\langle\hat{\varphi}^{r}\left|c_{0}^{-}\right| \hat{\varphi}_{s}\right\rangle=\delta_{s}^{r} \quad \Leftrightarrow \quad\left\langle\hat{\varphi}_{s}\left|c_{0}^{-}\right| \hat{\varphi}^{r}\right\rangle=\delta^{r}{ }_{s} .
$$

$\hat{\sigma}_{1}$ denotes the sign picked up while moving the vertex operators of $\left|\tilde{\phi}_{m_{1}+1}\right\rangle, \cdots\left|\tilde{\phi}_{m_{1}+m_{2}}\right\rangle$ through those of $\left|\hat{\phi}_{1}\right\rangle, \cdots\left|\hat{\phi}_{n_{1}-1}\right\rangle$ and $\hat{\sigma}_{2}$ is the sign picked up while moving the vertex operator for $\mathcal{X}_{0}\left|\hat{\varphi}^{r}\right\rangle$ through those of $\left|\tilde{\phi}_{m_{1}+1}\right\rangle, \cdots\left|\tilde{\phi}_{m_{1}+m_{2}}\right\rangle$. $\hat{\sigma}_{3}$ arises because we need to move $p_{2}$ of the $b$-ghost insertions associated with $\Omega_{p_{2}}^{\left(g_{2}, m_{2}, n_{2}\right)}$ through the vertex operators of $\left|\tilde{\phi}_{1}\right\rangle, \cdots\left|\tilde{\phi}_{m_{1}}\right\rangle,\left|\hat{\phi}_{1}\right\rangle \cdots\left|\hat{\phi}_{n_{1}-1}\right\rangle . \hat{\sigma}_{4}$ arises from the need to move the vertex operator of $\mathcal{X}_{0}\left|\hat{\varphi}^{r}\right\rangle$ through the $p_{2}$ insertions of $b$ ghosts associated with $\Omega_{p_{2}}^{\left(g_{2}, m_{2}, n_{2}\right)}$. Finally $\hat{\sigma}_{5}$ arises due to the need to move a factor of $b_{0}^{-}$through the $p_{1}$ insertions of $b$-ghost operators associated with $\Omega_{p_{1}}^{\left(g_{1}, m_{1}, n_{1}\right)}$ and the vertex operators of $\left|\tilde{\phi}_{1}\right\rangle, \cdots\left|\tilde{\phi}_{m_{1}}\right\rangle,\left|\hat{\phi}_{1}\right\rangle \cdots\left|\hat{\phi}_{n_{1}-1}\right\rangle$. 
The derivation of (3.10) follows in the same way as its NS-sector counterpart (3.8) described in $[1,10]$. The extra factor of $\mathcal{X}_{0}$ has its origin in the extra insertion of the PCO given in (3.2) involving plumbing fixture of Ramond punctures. Note that using (2.10) we could replace $\mathcal{X}_{0}$ by $\mathcal{G}$ in (3.10) and inserted a factor of $\mathcal{G}$ in front of $\left|\tilde{\varphi}^{r}\right\rangle$ in (3.8) to make the two equations look similar. This will be exploited later.

Generalization to type II string theories requires effectively 'doubling' the number of PCO's by including appropriate number of PCO's from the left-handed sector. Now for degenerations at NSR, RNS and RR punctures we insert respectively extra factor of $\mathcal{X}_{0}, \overline{\mathcal{X}}_{0}$ and $\mathcal{X}_{0} \overline{\mathcal{X}}_{0}$ around the punctures. Rest of the analysis proceeds in a straightforward manner.

\section{The 1PI effective field theory}

We shall now construct the gauge invariant equations of motion of a 1PI effective field theory whose off-shell amplitudes coincide with the ones constructed in section 3. Again for simplicity we first focus on the heterotic string theory. We shall begin by defining certain multilinear functions of the elements of $\mathcal{H}_{T}$ motivated by related construction in bosonic string field theory [38].

\subsection{The \{\} and [ ] products}

We define, for $\left|\Phi_{i}\right\rangle \in \mathcal{H}_{T}$, a function $\left\{\Phi_{1} \cdots \Phi_{N}\right\}$ as follows. ${ }^{5}$

1. $\left\{\Phi_{1} \cdots \Phi_{N}\right\}$ is a multilinear function of $\left|\Phi_{1}\right\rangle, \cdots\left|\Phi_{N}\right\rangle$ taking values in the grassmann algebra. Since we can express each $\left|\Phi_{i}\right\rangle$ as a linear combination of states in $\mathcal{H}_{(n)}$, it is enough to define $\left\{\Phi_{1} \cdots \Phi_{N}\right\}$ in the case where each $\left|\Phi_{i}\right\rangle$ is either an NS sector state or an R-sector state and has a fixed grassmannality.

2. $\left\{\Phi_{1} \cdots \Phi_{N}\right\}$ has the symmetry property

$$
\left\{\Phi_{1} \Phi_{2} \cdots \Phi_{i-1} \Phi_{i+1} \Phi_{i} \Phi_{i+2} \cdots \Phi_{N}\right\}=(-1)^{\gamma_{i} \gamma_{i+1}}\left\{\Phi_{1} \Phi_{2} \cdots \Phi_{N}\right\},
$$

where $\gamma_{i}$ is the grassmannality of $\left|\Phi_{i}\right\rangle$. Using this symmetry property we can bring all the NS sector states at the beginning of the set of $\left|\Phi_{i}\right\rangle$ 's. Thus it will be enough to define $\left\{\Phi_{1} \cdots \Phi_{N}\right\}$ for such an arrangement of the $\left|\Phi_{i}\right\rangle$ 's.

3. For $\left|\Phi_{1}\right\rangle, \cdots\left|\Phi_{m}\right\rangle \in \mathcal{H}_{N S}$ and $\left|\Phi_{m+1}\right\rangle, \cdots\left|\Phi_{m+n}\right\rangle \in \mathcal{H}_{R}$, we define

$$
\left\{\Phi_{1} \cdots \Phi_{m+n}\right\}=\sum_{g=0}^{\infty}\left(g_{s}\right)^{2 g} \int_{\mathcal{R}_{g, m, n}} \Omega_{6 g-6+2 m+2 n}^{(g, m, n)}\left(\left|\Phi_{1}\right\rangle, \cdots\left|\Phi_{m+n}\right\rangle\right) .
$$

Note that the property (4.1) under the exchange of an NS sector state with an R sector state is part of the definition of $\left\{\Phi_{1} \Phi_{2} \cdots \Phi_{N}\right\}$, whereas the same property under the exchange of two NS sector states or two R sector states follows from the property (3.6) of $\Omega_{p}^{(g, m, n)}$ and the fact that $\mathcal{R}_{g, m, n}$ is symmetric under the exchange of the NS-punctures and also under the exchange of the R-punctures.

\footnotetext{
${ }^{5}$ We only need the definitions of $\left\{\Phi_{1} \cdots \Phi_{N}\right\}$ and $\left[\Phi_{1} \cdots \Phi_{N}\right]$ in cases where each of the $\left|\Phi_{i}\right\rangle$ 's belong to $\mathcal{H}_{-1} \oplus \mathcal{H}_{-1 / 2}$ in heterotic string theory and $\mathcal{H}_{(-1,-1)} \oplus \mathcal{H}_{(-1 / 2,-1)} \oplus \mathcal{H}_{(-1,-1 / 2)} \oplus \mathcal{H}_{(-1 / 2,-1 / 2)}$ in type II string theories.
} 
It follows from the property of $\Omega_{p}^{(g, m, n)}$ that if the set $\left|\Phi_{1}\right\rangle, \cdots\left|\Phi_{N}\right\rangle$ contains $m$ NS sector and $n$ R-sector states then in order to get non-vanishing result for $\left\{\Phi_{1} \cdots \Phi_{N}\right\}$ we must have $\sum_{i=1}^{N} n_{i}=2 N$ and $\sum_{i=1}^{N} q_{i}=-m-n / 2$, where $\left(n_{i}, q_{i}\right)$ are the ghost and picture numbers of $\left|\Phi_{i}\right\rangle$. As a consequence of (3.4) and (3.7), (3.8), (3.10) we have the following important identity

$$
\begin{aligned}
& \sum_{i=1}^{N}(-1)^{\gamma_{1}+\cdots \gamma_{i-1}}\left\{\Phi_{1} \cdots \Phi_{i-1}\left(Q_{B} \Phi_{i}\right) \Phi_{i+1} \cdots \Phi_{N}\right\} \\
= & -\frac{1}{2} \sum_{\substack{\ell, k \geq 0 \\
\ell+k=N}} \sum_{\substack{\left\{i_{a} ; a=1, \cdots \ell\right\},\left\{j_{b} ; b=1, \cdots k\right\} \\
\{i a\} \cup\left\{j_{b}\right\}=\{1, \cdots N\}}} \sigma\left(\left\{i_{a}\right\},\left\{j_{b}\right\}\right)\left\{\Phi_{i_{1}} \cdots \Phi_{i_{\ell}} \varphi_{r}\right\}\left\{\left(\mathcal{G} \varphi^{r}\right) \Phi_{j_{1}} \cdots \Phi_{j_{k}}\right\}
\end{aligned}
$$

where $\sigma\left(\left\{i_{a}\right\},\left\{j_{b}\right\}\right)$ is the sign that one picks up while rearranging $b_{0}^{-}, \Phi_{1}, \cdots \Phi_{N}$ to $\Phi_{i_{1}}, \cdots \Phi_{i_{\ell}}, b_{0}^{-}, \Phi_{j_{1}}, \cdots \Phi_{j_{k}}$ and $\left\{\left|\varphi_{r}\right\rangle\right\}$ and $\left\{\left|\varphi^{r}\right\rangle\right\}$ are a set of dual basis of $\mathcal{H}_{T}$ satisfying

$$
\left\langle\varphi^{r}\left|c_{0}^{-}\right| \varphi_{s}\right\rangle=\delta^{r}{ }_{s} \quad \Leftrightarrow \quad\left\langle\varphi_{s}\left|c_{0}^{-}\right| \varphi^{r}\right\rangle=\delta^{r}{ }_{s},
$$

and the completeness relation

$$
\left|\varphi_{r}\right\rangle\left\langle\varphi^{r}|=| \varphi^{r}\right\rangle\left\langle\varphi_{r}\right|=b_{0}^{-} .
$$

Note the use of the symbol $\mathcal{G}$ defined in $(2.10)$ - it is identity if $\left|\varphi_{r}\right\rangle \in \mathcal{H}_{N S}$ and $\mathcal{X}_{0}$ if $\left|\varphi_{r}\right\rangle \in \mathcal{H}_{R}$. If $m$ of the $\left|\Phi_{i}\right\rangle$ 's represent NS sector states and $n=N-m$ of the $\left|\Phi_{i}\right\rangle$ 's represent $\mathrm{R}$ sector states then the coefficient of the $g_{s}{ }^{2 g}$ term on the left hand side of (4.3) is the integral of the left hand side of (3.4) over appropriate $\mathcal{R}_{g, m, n}$. On the other hand the coefficient of the $g_{s}{ }^{2 g}$ term on the right hand side of (4.3) represents the boundary terms that one obtains by integrating the total derivative term on the right hand side of (3.4) over $\mathcal{R}_{g, m, n}$. These boundary terms can be evaluated using (3.7) and the factorization properties (3.8), (3.10) yielding the expression given on the right hand side of (4.3). Special attention must be paid to the signs. The overall minus sign on the right hand side of (4.3) has its origin in the minus sign on the right hand side of (3.7). The $\sigma\left(\left\{i_{r}\right\},\left\{j_{s}\right\}\right)$ factor in (4.3) represents the product $\tilde{\sigma}_{1} \tilde{\sigma}_{5}$ or $\hat{\sigma}_{1} \hat{\sigma}_{5}$ in (3.8), (3.10). The $\tilde{\sigma}_{3}, \tilde{\sigma}_{4}$ factors in (3.8) and $\hat{\sigma}_{3}, \hat{\sigma}_{4}$ factors in (3.10) are unity since the degrees $p_{1}$ and $p_{2}$ of the differential forms are even. Finally the $\tilde{\sigma}_{2}$ factor in (3.8) and $\hat{\sigma}_{2}$ factor in (3.10) are not required in (4.3) since the $\left|\tilde{\varphi}_{r}\right\rangle$ and $\mathcal{X}_{0}\left|\hat{\varphi}^{r}\right\rangle$ factors which were in the 'incorrect positions' in these equations requiring this sign have been moved back to the 'correct position' sitting next to each other in (4.3).

Next we introduce a multilinear function $\left|\left[\Phi_{2} \cdots \Phi_{N}\right]\right\rangle \in \mathcal{H}_{T}$ of $(N-1)$ variables $\left|\Phi_{2}\right\rangle, \cdots\left|\Phi_{N}\right\rangle \in \mathcal{H}_{T}$, defined via the relations

$$
\left\langle\Phi_{1}\left|c_{0}^{-}\right|\left[\Phi_{2} \cdots \Phi_{N}\right]\right\rangle=\left\{\Phi_{1} \cdots \Phi_{N}\right\}
$$

for all $\left|\Phi_{1}\right\rangle \in \mathcal{H}_{T}$. Here $\langle A \mid B\rangle$ denotes the BPZ inner product. As in [1] we have dropped the ket symbol $\mid>$ from the states when they appear in the argument of \{\} or []. We shall also drop the ket symbol from $\left|\left[\Phi_{2} \cdots \Phi_{N}\right]\right\rangle$ except in inner products. If the set $\left|\Phi_{1}\right\rangle, \cdots\left|\Phi_{N}\right\rangle$ 
contains $m$ NS sector and $n$ R-sector states then $\left[\Phi_{2} \cdots \Phi_{N}\right]$ has ghost number equal to $3+\sum_{i=2}^{N} n_{i}-2(N-1)$ and picture number equal to $m+[n / 2]+\sum_{i=2}^{N} q_{i}-1$ where $[n / 2]$ denotes the largest integer $\leq n / 2$. In particular if all the NS sector states are in the -1 picture and all the $\mathrm{R}$-sector states are in the $-1 / 2$ picture then the picture number of $\left[\Phi_{2} \cdots \Phi_{N}\right]$ is -1 if $n$ is even and $-3 / 2$ if $n$ is odd.

Eq.(4.1) can now be translated to the identity

$$
\left[\Phi_{2} \cdots \Phi_{i-1} \Phi_{i+1} \Phi_{i} \Phi_{i+2} \cdots \Phi_{N}\right]=(-1)^{\gamma_{i} \gamma_{i+1}}\left[\Phi_{2} \cdots \Phi_{N}\right] .
$$

Furthermore (4.3) tells us that for $N \geq 1,{ }^{6}$

$$
\begin{aligned}
& Q_{B}\left[\Phi_{2} \cdots \Phi_{N}\right]+\sum_{i=2}^{N}(-1)^{\gamma_{2}+\cdots \gamma_{i-1}}\left[\Phi_{2} \cdots \Phi_{i-1}\left(Q_{B} \Phi_{i}\right) \Phi_{i+1} \cdots \Phi_{N}\right] \\
& =-\sum_{\substack{\ell, k \geq 0 \\
\ell+k=N-1}} \sum_{\substack{\left\{i_{a} ; a=1, \ldots \ell\right\},\left\{j_{b} ; b=1, \cdots k\right\} \\
\left\{i_{a}\right\} \cup\left\{j_{b}\right\}=\{2, \cdots N\}}} \sigma\left(\left\{i_{a}\right\},\left\{j_{b}\right\}\right)\left[\Phi_{i_{1}} \cdots \Phi_{i_{\ell}} \mathcal{G}\left[\Phi_{j_{1}} \cdots \Phi_{j_{k}}\right]\right]
\end{aligned}
$$

where in the last term the sum runs over all possible ways of splitting the set $\{2, \cdots N\}$ into the set $\left\{i_{a}\right\}$ and the set $\left\{j_{b}\right\} . \sigma\left(\left\{i_{a}\right\},\left\{j_{b}\right\}\right)$ is the sign that one picks up while rearranging $b_{0}^{-}, \Phi_{2}, \cdots \Phi_{N}$ to $\Phi_{i_{1}}, \cdots \Phi_{i_{\ell}}, b_{0}^{-}, \Phi_{j_{1}}, \cdots \Phi_{j_{k}}$. The inner product of (4.8) with an arbitrary state $\left\langle\Phi_{1}\right| c_{0}^{-}$is given by $(-1)^{\gamma_{1}}$ times (4.3). For the left hand sides the equality is obvious. For the right hand side, we note that in (4.3) we have two kinds of contributions: $\Phi_{1}$ can either be inside the first curly bracket or be inside the second curly bracket. These two contributions are identical due to the identity

$$
\left\{\Phi_{1} \cdots \Phi_{k} \varphi_{r}\right\}\left\{\left(\mathcal{G} \varphi^{r}\right) \widetilde{\Phi}_{1} \cdots \widetilde{\Phi}_{\ell}\right\}=(-1)^{\gamma+\tilde{\gamma}+\gamma \tilde{\gamma}}\left\{\widetilde{\Phi}_{1} \ldots \widetilde{\Phi}_{\ell} \varphi_{r}\right\}\left\{\left(\mathcal{G} \varphi^{r}\right) \Phi_{1} \cdots \Phi_{k}\right\}
$$

which we shall prove shortly. Here $\gamma$ is the total grassmannality of $\left|\Phi_{1}\right\rangle, \cdots\left|\Phi_{k}\right\rangle$ and $\tilde{\gamma}$ is the total grassmannality of $\left|\tilde{\Phi}_{1}\right\rangle, \cdots\left|\tilde{\Phi}_{\ell}\right\rangle$. Assuming this to be the case, we can only keep the terms on the right hand side of (4.3) where $\Phi_{1}$ is inside the first curly bracket and multiply the result by 2 . After being multiplied by $(-1)^{\gamma_{1}}$, this reproduces the inner product of $\left\langle\Phi_{1}\right| c_{0}^{-}$with the right hand side of (4.8).

Let us now prove (4.9). First we switch the order of the two terms on the right hand side of (4.9) to express this as

$$
\begin{aligned}
& \left\{\Phi_{1} \cdots \Phi_{k} \varphi_{r}\right\}\left\{\left(\mathcal{G} \varphi^{r}\right) \widetilde{\Phi}_{1} \ldots \widetilde{\Phi}_{\ell}\right\} \\
= & (-1)^{\gamma+\tilde{\gamma}+\gamma \tilde{\gamma}+\left(\gamma_{\varphi_{r}}+\tilde{\gamma}\right)\left(\gamma_{\varphi_{r}}+1+\gamma\right)}\left\{\left(\mathcal{G} \varphi^{r}\right) \Phi_{1} \ldots \Phi_{k}\right\}\left\{\widetilde{\Phi}_{1} \ldots \widetilde{\Phi}_{\ell} \varphi_{r}\right\},
\end{aligned}
$$

where $\gamma_{\varphi_{r}}, \gamma_{\varphi^{r}}$ are the grassmannalities of $\left|\varphi_{r}\right\rangle$ and $\left|\varphi^{r}\right\rangle$ and we have used $\gamma_{\varphi^{r}}=\gamma_{\varphi_{r}}+1$ mod 2. The latter relation follows from (4.4). Using (4.6), (4.1) we can express (4.10) as

$$
\begin{aligned}
& \left\langle\varphi_{r}\left|c_{0}^{-}\right|\left[\Phi_{1} \cdots \Phi_{k}\right]\right\rangle\left\langle\mathcal{G} \varphi^{r}\left|c_{0}^{-}\right|\left[\widetilde{\Phi}_{1} \ldots \widetilde{\Phi}_{\ell}\right]\right\rangle \\
= & \left\langle\mathcal{G} \varphi^{r}\left|c_{0}^{-}\right|\left[\Phi_{1} \cdots \Phi_{k}\right]\right\rangle\left\langle\varphi_{r}\left|c_{0}^{-}\right|\left[\widetilde{\Phi}_{1} \cdots \widetilde{\Phi}_{\ell}\right]\right\rangle(-1)^{\gamma+\tilde{\gamma}+\gamma \tilde{\gamma}+\left(\gamma_{\varphi_{r}}+\tilde{\gamma}\right)\left(\gamma_{\varphi_{r}}+1+\gamma\right)+\gamma_{\varphi_{r}}(\gamma+\tilde{\gamma})} .
\end{aligned}
$$

\footnotetext{
${ }^{6}$ Note that inside $[\cdots]$ in the first term of $(4.8)$ the first argument is $\Phi_{2}$ and hence there are only $N-1$ arguments. Thus for $N=1$ we have the equation $Q_{B}[]+[\mathcal{G}[]]=0$.
} 
Now we have

$$
\left\langle A\left|c_{0}^{-}\right| B\right\rangle=(-1)^{\gamma_{A}+\gamma_{B}+\gamma_{A} \gamma_{B}+1}\left\langle B\left|c_{0}^{-}\right| A\right\rangle, \quad\left\langle\mathcal{G} A\left|c_{0}^{-}\right| B\right\rangle=(-1)^{\gamma_{A}+\gamma_{B}+\gamma_{A} \gamma_{B}+1}\left\langle B\left|c_{0}^{-} \mathcal{G}\right| A\right\rangle .
$$

Applying the first equation on the first term on the left hand side of (4.11) and the second equation on the first term on the right hand side of (4.11), and noting that the grassmannality of $\left[\Phi_{1} \cdots \Phi_{k}\right]$ is $\gamma+1 \bmod 2$, we can express (4.11) as

$$
\begin{aligned}
& \left\langle\left[\Phi_{1} \cdots \Phi_{k}\right]\left|c_{0}^{-}\right| \varphi_{r}\right\rangle\left\langle\mathcal{G} \varphi^{r}\left|c_{0}^{-}\right|\left[\widetilde{\Phi}_{1} \cdots \widetilde{\Phi}_{\ell}\right]\right\rangle \\
& =(-1)^{\gamma+\tilde{\gamma}+\gamma \tilde{\gamma}+\left(\gamma_{\varphi_{r}}+\tilde{\gamma}\right)\left(\gamma_{\varphi_{r}}+1+\gamma\right)+\gamma_{\varphi_{r}}(\gamma+\tilde{\gamma})+\gamma_{\varphi_{r}} \gamma+\left(\gamma_{\varphi_{r}}+1\right) \gamma} \\
& \times\left\langle\left[\Phi_{1} \cdots \Phi_{k}\right]\left|c_{0}^{-} \mathcal{G}\right| \varphi^{r}\right\rangle\left\langle\varphi_{r}\left|c_{0}^{-}\right|\left[\widetilde{\Phi}_{1} \ldots \widetilde{\Phi}_{\ell}\right]\right\rangle .
\end{aligned}
$$

Using the completeness relations $(4.5)$, the fact that $\left[\mathcal{G}, c_{0}^{-}\right]$vanishes when sandwiched between states annihilated by $b_{0}^{-}$, and simplifying the exponent of $(-1),(4.13)$ reduces to

$$
\left\langle\left[\Phi_{1} \cdots \Phi_{k}\right]\left|c_{0}^{-} \mathcal{G}\right|\left[\widetilde{\Phi}_{1} \cdots \widetilde{\Phi}_{\ell}\right]\right\rangle=\left\langle\left[\Phi_{1} \cdots \Phi_{k}\right]\left|c_{0}^{-} \mathcal{G}\right|\left[\widetilde{\Phi}_{1} \ldots \widetilde{\Phi}_{\ell}\right]\right\rangle
$$

which is an identity. This in turn proves (4.9).

\subsection{The equation of motion and its gauge invariance}

A general string field configuration is taken to be an element $|\Psi\rangle$ of $\mathcal{H}_{(-1)} \oplus \mathcal{H}_{(-1 / 2)}$ of ghost number 2 , with the component along $\mathcal{H}_{(-1)}$ representing the bosonic fields and the component along $\mathcal{H}_{(-1 / 2)}$ representing the fermionic fields. Thus $|\Psi\rangle$ is grassmann even. The equations of motion for $|\Psi\rangle$ in the 1PI effective heterotic string field theory is taken to be $\mathrm{b}^{7}$

$$
|\mathcal{E}\rangle=0, \quad|\mathcal{E}\rangle \equiv Q_{B}|\Psi\rangle+\sum_{n=1}^{\infty} \frac{1}{(n-1) !} \mathcal{G}\left[\Psi^{n-1}\right] .
$$

Note that $Q_{B}|\Psi\rangle$ is an element of $\mathcal{H}_{(-1)}+\mathcal{H}_{(-1 / 2)}$ of ghost number 3 whereas $\left[\Psi^{n-1}\right]$ is an element of $\mathcal{H}_{(-1)}+\mathcal{H}_{(-3 / 2)}$ of ghost number 3. The operation of $\mathcal{G}$ is essential to map the latter to an element of $\mathcal{H}_{(-1)}+\mathcal{H}_{(-1 / 2)}$. The infinitesimal gauge transformation is generated by an element $|\Lambda\rangle$ of $\mathcal{H}_{(-1)} \oplus \mathcal{H}_{(-1 / 2)}$ of ghost number 1. $|\Lambda\rangle$ is grassmann odd. The gauge transformation law of $|\Psi\rangle$ is

$$
|\delta \Psi\rangle=Q_{B}|\Lambda\rangle+\sum_{n=0}^{\infty} \frac{1}{n !} \mathcal{G}\left[\Psi^{n} \Lambda\right]
$$

Again the operation of $\mathcal{G}$ is crucial in bringing $\left[\Psi^{n} \Lambda\right]$ which is an element of $\mathcal{H}_{(-1)}+\mathcal{H}_{(-3 / 2)}$ to an element of $\mathcal{H}_{(-1)} \oplus \mathcal{H}_{(-1 / 2)}$.

\footnotetext{
${ }^{7}$ Alternatively we could take the string field to be an element $|\widetilde{\Psi}\rangle \in \mathcal{H}_{(-1)} \oplus \mathcal{H}_{(-3 / 2)}$ of ghost number 2 , and write the equation of motion as $Q_{B}|\widetilde{\Psi}\rangle+\sum_{n=1}^{\infty} \frac{1}{(n-1) !}\left[(\mathcal{G} \widetilde{\Psi})^{n-1}\right]=0 .|\widetilde{\Psi}\rangle$ and $|\Psi\rangle$ will be related as $|\Psi\rangle=\mathcal{G}|\widetilde{\Psi}\rangle$. Since the cohomology of $Q_{B}$ in picture numbers $-1 / 2$ and $-3 / 2$ coincide [11], this will give a sensible set of equations of motion. We shall not explore this in detail, but the reader will find some related comments at the end of section 4.3 .
} 
We shall now show that the equations of motion are gauge covariant, i.e. if $|\Psi\rangle$ satisfies equations of motion then its gauge transform also satisfies equations of motion. Taking the gauge variation of $(4.15)$ gives

$$
|\delta \mathcal{E}\rangle=Q_{B}|\delta \Psi\rangle+\sum_{n=1}^{\infty} \frac{1}{(n-1) !} \mathcal{G}\left[\Psi^{n-1} \delta \Psi\right] .
$$

Our goal is to show that $|\delta \mathcal{E}\rangle$ vanishes when $|\mathcal{E}\rangle$ vanishes. Now using (4.16) we can express (4.17) as

$$
|\delta \mathcal{E}\rangle=\sum_{n=0}^{\infty} \frac{1}{n !} Q_{B} \mathcal{G}\left[\Psi^{n} \Lambda\right]+\sum_{n=1}^{\infty} \frac{1}{(n-1) !} \mathcal{G}\left[\Psi^{n-1} Q_{B} \Lambda\right]+\sum_{n=1}^{\infty} \frac{1}{(n-1) !} \sum_{m=0}^{\infty} \frac{1}{m !} \mathcal{G}\left[\Psi^{n-1} \mathcal{G}\left[\Psi^{m} \Lambda\right]\right]
$$

We now manipulate the first term on the right hand side using $\left[Q_{B}, \mathcal{G}\right]=0$ and (4.8). Since $|\Psi\rangle$ is grassmann even and $|\Lambda\rangle$ is grassmann odd, we get

$$
\begin{aligned}
|\delta \mathcal{E}\rangle= & -\sum_{n=1}^{\infty} \frac{1}{(n-1) !} \mathcal{G}\left[\Psi^{n-1}\left(Q_{B} \Psi\right) \Lambda\right]-\sum_{n=0}^{\infty} \frac{1}{n !} \mathcal{G}\left[\Psi^{n} Q_{B} \Lambda\right]-\sum_{p=0}^{\infty} \frac{1}{p !} \sum_{m=0}^{\infty} \frac{1}{m !} \mathcal{G}\left[\Psi^{p} \mathcal{G}\left[\Psi^{m} \Lambda\right]\right] \\
& +\sum_{p=0}^{\infty} \frac{1}{p !} \sum_{m=0}^{\infty} \frac{1}{m !} \mathcal{G}\left[\Psi^{p} \Lambda \mathcal{G}\left[\Psi^{m}\right]\right] \\
& +\sum_{n=1}^{\infty} \frac{1}{(n-1) !} \mathcal{G}\left[\Psi^{n-1} Q_{B} \Lambda\right]+\sum_{n=1}^{\infty} \frac{1}{(n-1) !} \sum_{m=0}^{\infty} \frac{1}{m !} \mathcal{G}\left[\Psi^{n-1} \mathcal{G}\left[\Psi^{m} \Lambda\right]\right]
\end{aligned}
$$

The first and the fourth term on the right hand side cancel using the equations of motion (4.15). The second and fifth terms cancel and the third and the sixth terms cancel. Thus we get

$$
|\delta \mathcal{E}\rangle=0
$$

This proves that the equations of motion transform covariantly under gauge transformations.

Note that if we restrict to the states in the NS sector then $\mathcal{G}$ can be replaced by the identity operator and the equations of motion reduce to those which were derived from the 1PI action in [1]. However once we include the R-sector states there is no fully satisfactory action from which the equations of motion (4.15) can be derived. More discussion on this can be found in section 4.3 .

\subsection{Auxiliary action and S-matrix elements}

We shall now argue that the tree level Green's functions computed from the 1PI effective theory described above reproduces the off-shell amplitudes described in section 3 . In that case the S-matrix elements computed via LSZ prescription from these two approaches would also agree. Although it is in principle possible to compute the tree level S-matrix from the equations of motion directly we shall take a short-cut by using an action with additional states from which the equations of motion can be derived. For this we introduce a new set 
of fields $|\widetilde{\Psi}\rangle \in \mathcal{H}_{(-1)} \oplus \mathcal{H}_{(-3 / 2)}$ of ghost number 2 and consider the action

$$
S=g_{s}{ }^{-2}\left[-\frac{1}{2}\left\langle\widetilde{\Psi}\left|c_{0}^{-} Q_{B} \mathcal{G}\right| \widetilde{\Psi}\right\rangle+\left\langle\widetilde{\Psi}\left|c_{0}^{-} Q_{B}\right| \Psi\right\rangle+\sum_{n=0}^{\infty} \frac{1}{n !}\left\{\Psi^{n}\right\}\right] .
$$

The equation of motion for $|\widetilde{\Psi}\rangle$ derived from (4.21) is

$$
Q_{B}(|\Psi\rangle-\mathcal{G}|\widetilde{\Psi}\rangle)=0
$$

On the other hand the equation of motion of $|\Psi\rangle$ is

$$
Q_{B}|\widetilde{\Psi}\rangle+\sum_{n=1}^{\infty} \frac{1}{(n-1) !}\left[\Psi^{n-1}\right]=0 .
$$

Applying $\mathcal{G}$ on (4.23) and using (4.22) we recover the equation of motion (4.15) of $|\Psi\rangle$. It is easy to see that the action (4.21) is invariant under the infinitesimal gauge transformation (4.16) if we also transform $|\widetilde{\Psi}\rangle$ as

$$
|\delta \widetilde{\Psi}\rangle=Q_{B}|\widetilde{\Lambda}\rangle+\sum_{n=0}^{\infty} \frac{1}{n !}\left[\Psi^{n} \Lambda\right],
$$

where $|\widetilde{\Lambda}\rangle \in \mathcal{H}_{(-1)} \oplus \mathcal{H}_{(-3 / 2)}$ and carries ghost number 1 .

We can now gauge fix the theory in the Siegel gauge $b_{0}^{+}|\Psi\rangle=0, b_{0}^{+}|\widetilde{\Psi}\rangle=0$ in which case the kinetic term in the $(|\widetilde{\Psi}\rangle,|\Psi\rangle)$ space is proportional to

$$
c_{0}^{-} c_{0}^{+} L_{0}^{+}\left(\begin{array}{cc}
-\mathcal{G} & 1 \\
1 & 0
\end{array}\right)
$$

leading to the propagator

$$
b_{0}^{+} b_{0}^{-}\left(L_{0}^{+}\right)^{-1} \delta_{L_{0}, \bar{L}_{0}}\left(\begin{array}{ll}
0 & 1 \\
1 & \mathcal{G}
\end{array}\right) .
$$

Since the interaction terms involve only the field $|\Psi\rangle$, only the $|\Psi\rangle$ propagator is relevant for computing the Green's functions in the Siegel gauge with external legs truncated. This is proportional to $b_{0}^{-} b_{0}^{+}\left(L_{0}^{+}\right)^{-1} \delta_{L_{0}, \bar{L}_{0}} \mathcal{G}$ which is precisely the propagator used in the analysis of section 3. Standard argument then shows that the off-shell Green's functions with external tree level propagator truncated, and only $|\Psi\rangle$ as external states, coincide with the off-shell amplitudes described in section 3, with the 1PI contribution reproducing the part of the integration cycle that is described by $\mathcal{R}_{g, m, n}$ and the one particle reducible (1PR) contributions reproducing the rest of the components of the integration cycle. Together they describe the full integration cycle whose projection on the base covers the whole of $\mathcal{M}_{g, m, n}$. Thus the S-matrix elements computed from these Green's functions will also agree with the ones computed from the off-shell amplitudes described in section 3 .

Could we use the action (4.21) for defining the 1PI effective theory? The problem with this is that the equations of motion (4.22), (4.23) have more classical solutions than the ones expected in string theory. For example at the linearized level we can consider 
solutions with $|\widetilde{\Psi}\rangle=0, Q_{B}|\Psi\rangle=0$, and independently another set of solutions for which $|\Psi\rangle=0, Q_{B}|\widetilde{\Psi}\rangle=0$. This will double the number of physical states. This does not make any difference as long as we are using this action to compute tree level S-matrix elements with external $|\Psi\rangle$ states (which could in principle be computed just from the equations of motion (4.15) of $|\Psi\rangle$ ), but due to these extra states the action (4.21) cannot be regarded as the fundamental action for describing the 1PI effective string field theory. We could try to remove the extra states by adding a constraint $|\Psi\rangle=\mathcal{G}|\widetilde{\Psi}\rangle$. Now (4.22) holds automatically and (4.23) reproduces the equations of motion described in footnote 7, but this constraint has to be imposed externally and does not follow from the action. On the other hand if we use this constraint to eliminate $|\Psi\rangle$ from the action (4.21) and treat $|\widetilde{\Psi}\rangle$ as independent field variables, then we arrive at the action

$$
g_{s}{ }^{-2}\left[\frac{1}{2}\left\langle\widetilde{\Psi}\left|c_{0}^{-} Q_{B} \mathcal{G}\right| \widetilde{\Psi}\right\rangle+\sum_{n=1}^{\infty} \frac{1}{n !}\left\{(\mathcal{G} \widetilde{\Psi})^{n}\right\}\right] .
$$

Now the kinetic operator becomes proportional to $c_{0}^{-} Q_{B} \mathcal{G}$ which may have additional zeroes from the kernel of $\mathcal{G}$ and hence the spectrum of physical states in this theory will again differ from the expected spectrum of string theory.

\subsection{Generalizaton to type II string theories}

Generalization of the above analysis to type II string theories is straightforward. The only difference is that the string field is now taken to be a grassmann even element of $\mathcal{H}_{(-1,-1)} \oplus \mathcal{H}_{(-1,-1 / 2)} \oplus \mathcal{H}_{(-1 / 2,-1)} \oplus \mathcal{H}_{(-1 / 2,-1 / 2)}$ carrying ghost number 2. However the use of the symbol $\mathcal{G}$ - now defined as in (2.14) - ensures that all the formulæ derived for the heterotic string theory continue to be valid for type II string theories. The analysis of section 4.3 can also be extended to this case by taking $|\widetilde{\Psi}\rangle \in \mathcal{H}_{(-1,-1)} \oplus \mathcal{H}_{(-1,-3 / 2)} \oplus$ $\mathcal{H}_{(-3 / 2,-1)} \oplus \mathcal{H}_{(-3 / 2,-3 / 2)}$.

\section{Effect of changing the local coordinates and/or PCO locations}

We now study the effect of changing the choice of local coordinates and/or the locations of the PCO's on the 1PI effective theory following [43]. A change of this form will correspond to a new choice of the $(6 g-6+2 m+2 n)$ dimensional subspaces $\mathcal{R}_{g, m, n}$ in $\widetilde{\mathcal{P}}_{g, m, n}$ satisfying (3.7). Let us denote them by $\mathcal{R}_{g, m, n}^{\prime}$. We shall consider infinitesimal deformations so that $\mathcal{R}_{g, m, n}$ and $\mathcal{R}_{g, m, n}^{\prime}$ are close in $\widetilde{\mathcal{P}}_{g, m, n}$ and denote the corresponding change in $|\mathcal{E}\rangle$ defined in (4.15) by $|\widehat{\delta} \mathcal{E}\rangle$. Our goal will be to show that there is a possible field redefinition $|\Psi\rangle \rightarrow|\Psi\rangle+|\tilde{\delta} \Psi\rangle$ such that the change $|\tilde{\delta} \mathcal{E}\rangle$ in $|\mathcal{E}\rangle$ induced by this field redefinition reproduces $|\widehat{\delta} \mathcal{E}\rangle$ upon using equations of motion, i.e.

$$
|\widehat{\delta} \mathcal{E}\rangle-|\tilde{\delta} \mathcal{E}\rangle=0
$$

when $|\mathcal{E}\rangle=0$. This will imply that the effect of the change in local coordinates / PCO locations can be compensated by a field redefinition. 
To proceed, let us decompose the string field $|\Psi\rangle$ into its NS and R part:

$$
|\Psi\rangle=\left|\Psi_{N S}\right\rangle+\left|\Psi_{R}\right\rangle
$$

so that we can write

$$
|\mathcal{E}\rangle=Q_{B}\left(\left|\Psi_{N S}\right\rangle+\left|\Psi_{R}\right\rangle\right)+\sum_{m, n=0}^{\infty} \frac{1}{m ! n !} \mathcal{G}\left[\left(\Psi_{N S}\right)^{m}\left(\Psi_{R}\right)^{n}\right] .
$$

This gives, using (4.6),

$$
\left\langle\Phi\left|c_{0}^{-}\right| \mathcal{E}\right\rangle=\left\langle\Phi \left| c_{0}^{-} Q_{B}\left(\left|\Psi_{N S}\right\rangle+\left|\Psi_{R}\right\rangle\right)+\sum_{m, n=0}^{\infty} \frac{1}{m ! n !}\left\{(\mathcal{G} \Phi)\left(\Psi_{N S}\right)^{m}\left(\Psi_{R}\right)^{n}\right\},\right.\right.
$$

for any state $|\Phi\rangle \in \mathcal{H}_{(-1)} \oplus \mathcal{H}_{(-3 / 2)}$ of ghost number 2. We shall take $|\Phi\rangle$ to be grassmann even for convenience, but the analysis can be repeated for grassmann odd $|\Phi\rangle$ as well. Decomposing $|\Phi\rangle$ as $\left|\Phi_{N S}\right\rangle+\left|\Phi_{R}\right\rangle$ we get, using (4.2) and (5.4)

$$
\begin{aligned}
& \left\langle\Phi\left|c_{0}^{-}\right| \widehat{\delta} \mathcal{E}\right\rangle \\
= & \sum_{g=0}^{\infty} g_{s}{ }^{2 g} \sum_{m, n=0}^{\infty} \frac{1}{m ! n !}\left[\left(\int_{\mathcal{R}_{g, m+1, n}^{\prime}}-\int_{\mathcal{R}_{g, m+1, n}}\right) \Omega_{6 g-6+2 m+2 n+2}^{(g, m+1, n)}\left(\mathcal{G}\left|\Phi_{N S}\right\rangle,\left|\Psi_{N S}\right\rangle^{\otimes m},\left|\Psi_{R}\right\rangle^{\otimes n}\right)\right] \\
& +\sum_{g=0}^{\infty} g_{s}{ }^{2 g} \sum_{m, n=0}^{\infty} \frac{1}{m ! n !}\left[\left(\int_{\mathcal{R}_{g, m, n+1}^{\prime}}-\int_{\mathcal{R}_{g, m, n+1}}\right) \Omega_{6 g-6+2 m+2 n+2}^{(g, m, n+1)}\left(\left|\Psi_{N S}\right\rangle^{\otimes m}, \mathcal{G}\left|\Phi_{R}\right\rangle,\left|\Psi_{R}\right\rangle^{\otimes n}\right)\right]
\end{aligned}
$$

where $|\Psi\rangle^{\otimes n}$ denotes that there are $n$ entries of $|\Psi\rangle$ in the argument.

Let us first focus on the part involving $\left|\Phi_{N S}\right\rangle$ so that we only have the first term on the right hand side of (5.5). Let $\widehat{U}_{g, m, n}$ be an infinitesimal vector field that takes a point in $\mathcal{R}_{g, m, n}$ to a neighbouring point in $\mathcal{R}_{g, m, n}^{\prime} . \widehat{U}_{g, m, n}$ is defined up to addition of tangent vectors of $\mathcal{R}_{g, m, n}$. In this case the part of (5.5) involving $\left|\Phi_{N S}\right\rangle$ can be expressed as $[1,43]$

$$
\begin{aligned}
& \left\langle\Phi_{N S}\left|c_{0}^{-}\right| \widehat{\delta} \mathcal{E}\right\rangle \\
= & \sum_{g=0}^{\infty} g_{s}{ }^{2 g} \sum_{m, n=0}^{\infty} \frac{1}{m ! n !}\left[\int_{\mathcal{R}_{g, m+1, n}} d \Omega_{6 g-6+2 m+2 n+2}^{(g, m+1, n)}\left[\widehat{U}_{g, m+1, n}\right]\left(\mathcal{G}\left|\Phi_{N S}\right\rangle,\left|\Psi_{N S}\right\rangle^{\otimes m},\left|\Psi_{R}\right\rangle^{\otimes n}\right)\right. \\
& \left.+\int_{\partial \mathcal{R}_{g, m+1, n}} \Omega_{6 g-6+2 m+2 n+2}^{(g, m+1, n)}\left[\widehat{U}_{g, m+1, n}\right]\left(\mathcal{G}\left|\Phi_{N S}\right\rangle,\left|\Psi_{N S}\right\rangle^{\otimes m},\left|\Psi_{R}\right\rangle^{\otimes n}\right)\right]
\end{aligned}
$$

where for any $p$-form $\omega_{p}, \omega_{p}[\widehat{U}]$ denotes the contraction of $\omega_{p}$ with the vector field $\widehat{U}$ :

$$
\omega_{i_{1} \cdots i_{p}} d y^{i_{1}} \wedge \cdots \wedge d y^{i_{p}}[\widehat{U}] \equiv \widehat{U}^{i_{1}} \omega_{i_{1} i_{2} \cdots i_{p}} d y^{i_{2}} \wedge \cdots \wedge d y^{i_{p}}
$$

An intuitive understanding of (5.6) can be found in figure 1 of [1]. We manipulate the first term on the right hand side of (5.6) using (3.4) and the second term using (3.8) and (3.10). The second term can be further simplified by noting that on $\partial \mathcal{R}_{g, m+1, n}$ - which can be regarded as the result of plumbing fixture of two 1PI Riemann surfaces with $s=0$ - the state $\mathcal{G}\left|\Phi_{N S}\right\rangle$ can be inserted either on the first surface or on the second surface. Since 
these contributions are equal, we shall insert $\mathcal{G}\left|\Phi_{N S}\right\rangle$ on the first surface and multiply the result by a factor of two. Furthermore on $\partial \mathcal{R}_{g, m+1, n}$ the vector field $\widehat{U}_{g, m, n}$ reduces to the sum of two vector fields labelling the deformations of the choice of local coordinates and PCO locations on the two components that are glued to produce $\partial \mathcal{R}_{g, m+1, n}$. This gives

$$
\begin{aligned}
& \left\langle\Phi_{N S}\left|c_{0}^{-}\right| \widehat{\delta} \mathcal{E}\right\rangle \\
& =\sum_{g=0}^{\infty} g_{s}{ }^{2 g}\left[-\sum_{m, n=0}^{\infty} \frac{1}{m ! n !} \int_{\mathcal{R}_{g, m+1, n}} \Omega_{6 g-6+2 m+2 n+3}^{(g, m+1, n)}\left[\widehat{U}_{g, m+1, n}\right]\left(Q_{B} \mathcal{G}\left|\Phi_{N S}\right\rangle,\left|\Psi_{N S}\right\rangle^{\otimes m},\left|\Psi_{R}\right\rangle^{\otimes n}\right)\right. \\
& -\sum_{m=1}^{\infty} \sum_{n=0}^{\infty} \frac{1}{(m-1) ! n !} \int_{\mathcal{R}_{g, m+1, n}} \Omega_{6 g-6+2 m+2 n+3}^{(g, m+1, n)}\left[\widehat{U}_{g, m+1, n}\right]\left(\mathcal{G}\left|\Phi_{N S}\right\rangle, Q_{B}\left|\Psi_{N S}\right\rangle,\left|\Psi_{N S}\right\rangle^{\otimes(m-1)},\left|\Psi_{R}\right\rangle^{\otimes n}\right) \\
& \left.-\sum_{m=0}^{\infty} \sum_{n=1}^{\infty} \frac{1}{m !(n-1) !} \int_{\mathcal{R}_{g, m+1, n}} \Omega_{6 g-6+2 m+2 n+3}^{(g, m+1, n)}\left[\widehat{U}_{g, m+1, n}\right]\left(\mathcal{G}\left|\Phi_{N S}\right\rangle,\left|\Psi_{N S}\right\rangle^{\otimes m}, Q_{B}\left|\Psi_{R}\right\rangle,\left|\Psi_{R}\right\rangle^{\otimes(n-1)}\right)\right] \\
& -\sum_{g_{1}, g_{2}} g_{s}^{2\left(g_{1}+g_{2}\right)} \sum_{m_{1}, m_{2}, n_{1}, n_{2} \geq 0} \frac{1}{m_{1} ! m_{2} ! n_{1} ! n_{2} !} \int_{\left\{\mathcal{R}_{g_{1}, m_{1}+2, n_{1}}, \mathcal{R}_{\left.g_{2}, m_{2}+1, n_{2}\right\}}\right.} \\
& \Omega_{6\left(g_{1}+g_{2}, m_{1}\right)-6+2\left(m_{1}+m_{2}+m_{2}+n_{1}+n_{2}+1\right)}^{\left(g_{1}\right)}\left[\widehat{U}_{g_{1}, m_{1}+2, n_{1}}+\widehat{U}_{g_{2}, m_{2}+1, n_{2}}\right]\left(\mathcal{G}\left|\Phi_{N S}\right\rangle,\left|\Psi_{N S}\right\rangle^{\otimes\left(m_{1}+m_{2}\right)},\left|\Psi_{R}\right\rangle^{\otimes\left(n_{1}+n_{2}\right)}\right) \\
& -\sum_{g_{1}, g_{2}} g_{s}{ }^{2\left(g_{1}+g_{2}\right)} \sum_{m_{1}, m_{2}, n_{1}, n_{2} \geq 0} \frac{1}{m_{1} ! m_{2} ! n_{1} ! n_{2} !} \int_{\left\{\mathcal{R}_{g_{1}, m_{1}+1, n_{1}+1} ; \mathcal{R}_{\left.g_{2}, m_{2}, n_{2}+1\right\}}\right.} \\
& \Omega_{6\left(g_{1}+g_{2}, m_{1}\right)-6+2\left(m_{1}+m_{1}+m_{2}+n_{1}+n_{2}+1\right)}^{\left(g_{1}\right)}\left[\widehat{U}_{g_{1}, m_{1}+1, n_{1}+1}+\widehat{U}_{g_{2}, m_{2}, n_{2}+1}\right]\left(\mathcal{G}\left|\Phi_{N S}\right\rangle,\left|\Psi_{N S}\right\rangle^{\otimes\left(m_{1}+m_{2}\right)},\left|\Psi_{R}\right\rangle^{\otimes\left(n_{1}+n_{2}\right)}\right) \\
& \equiv I_{1}+I_{2}+I_{3}+I_{4}+I_{5},
\end{aligned}
$$

where $I_{1}, \cdots I_{5}$ denote the five terms appearing on the right hand side. In the expressions for $I_{4}$ and $I_{5}$ it is understood that $\mathcal{G}\left|\Phi_{N S}\right\rangle, m_{1}$ of the $\left|\Psi_{N S}\right\rangle$ 's and $n_{1}$ of the $\left|\Psi_{R}\right\rangle$ 's are inserted on the first Riemann surface and $m_{2}$ of the $\left|\Psi_{N S}\right\rangle$ 's and $n_{2}$ of the $\left|\Psi_{R}\right\rangle$ 's are inserted on the second Riemann surface. In the first three terms the minus signs have their origin in the $(-1)^{p}$ factor in (3.4). In the last two terms the minus signs come from the application of (3.7). The terms involving $\left|\Phi_{R}\right\rangle$ can be analyzed in an identical manner, with the minus signs remaining the same.

We shall now show that the change in $|\mathcal{E}\rangle$ given in (5.8) together with its counterpart involving $\left|\Phi_{R}\right\rangle$ can be regarded as the result of a redefinition of the field $|\Psi\rangle$ to $|\Psi\rangle+|\tilde{\delta} \Psi\rangle$ in the sense of (5.1) if we take $|\tilde{\delta} \Psi\rangle$ to be of the form

$$
\begin{aligned}
& \left\langle\phi\left|c_{0}^{-}\right| \tilde{\delta} \Psi\right\rangle \\
= & -\sum_{g=0}^{\infty} g_{s}{ }^{2 g} \sum_{m, n=0}^{\infty} \frac{1}{m ! n !} \int_{\mathcal{R}_{g, m+1, n}} \Omega_{6 g-5+2 m+2 n+2}^{(g, m+1, n)}\left[\widehat{U}_{g, m+1, n}\right]\left(\mathcal{G}\left|\phi_{N S}\right\rangle,\left|\Psi_{N S}\right\rangle^{\otimes m},\left|\Psi_{R}\right\rangle^{\otimes n}\right) \\
& -\sum_{g=0}^{\infty} g_{s}{ }^{2 g} \sum_{m, n=0}^{\infty} \frac{1}{m ! n !} \int_{\mathcal{R}_{g, m, n+1}} \Omega_{6 g-5+2 m+2 n+2}^{(g, m, n+1)}\left[\widehat{U}_{g, m, n+1}\right]\left(\left|\Psi_{N S}\right\rangle^{\otimes m}, \mathcal{G}\left|\phi_{R}\right\rangle,\left|\Psi_{R}\right\rangle^{\otimes n}\right),
\end{aligned}
$$

for any grassmann odd ${ }^{8}$ state $|\phi\rangle=\left|\phi_{N S}\right\rangle+\left|\phi_{R}\right\rangle \in \mathcal{H}_{T}$. Now, using (4.15) we get

$$
\left\langle\Phi\left|c_{0}^{-}\right| \tilde{\delta} \mathcal{E}\right\rangle=\left\langle\Phi\left|c_{0}^{-} Q_{B}\right| \tilde{\delta} \Psi\right\rangle+\sum_{n=1}^{\infty} \frac{1}{(n-1) !}\left\langle\Phi\left|c_{0}^{-} \mathcal{G}\right|\left[\Psi^{n-1} \tilde{\delta} \Psi\right]\right\rangle
$$

\footnotetext{
${ }^{8}$ The result for grassmann even state can be read out by multiplying both sides of (5.9) by a grassmann odd number and moving it through various factors so that it multiplies $|\phi\rangle$. This gives extra minus signs in both terms on the right hand side of (5.9) since we have to move the grassmann number through the $6 g-5+2 m+2 n+2$ insertions of $b$-ghost field associated with $\Omega_{6 g-5+2 m+2 n+2}^{(g, m+1, n)}$.
} 


$$
\begin{aligned}
& =\left\langle\left(Q_{B} \Phi\right)\left|c_{0}^{-}\right| \tilde{\delta} \Psi\right\rangle+\sum_{n=1}^{\infty} \frac{1}{(n-1) !}\left\{(\mathcal{G} \Phi) \Psi^{n-1} \tilde{\delta} \Psi\right\} \\
& =\left\langle\left(Q_{B} \Phi\right)\left|c_{0}^{-}\right| \tilde{\delta} \Psi\right\rangle+\sum_{n=1}^{\infty} \frac{1}{(n-1) !}\left\langle\left[(\mathcal{G} \Phi) \Psi^{n-1}\right]\left|c_{0}^{-}\right| \tilde{\delta} \Psi\right\rangle
\end{aligned}
$$

Using (5.9) we can express this as

$$
\begin{aligned}
& -\sum_{g=0}^{\infty} g_{s}{ }^{2 g} \sum_{m, n=0}^{\infty} \frac{1}{m ! n !} \int_{\mathcal{R}_{g, m+1, n}} \Omega_{6 g-5+2 m+2 n+2}^{(g, m+1, n)}\left[\widehat{U}_{g, m+1, n}\right]\left(Q_{B} \mathcal{G}\left|\Phi_{N S}\right\rangle,\left|\Psi_{N S}\right\rangle^{\otimes m},\left|\Psi_{R}\right\rangle^{\otimes n}\right) \\
& -\sum_{g=0}^{\infty} g_{s}{ }^{2 g} \sum_{m, n=0}^{\infty} \frac{1}{m ! n !} \int_{\mathcal{R}_{g, m, n+1}} \Omega_{6 g-5+2 m+2 n+2}^{(g, m, n+1)}\left[\widehat{U}_{g, m, n+1}\right]\left(\left|\Psi_{N S}\right\rangle^{\otimes m}, Q_{B} \mathcal{G}\left|\Phi_{R}\right\rangle,\left|\Psi_{R}\right\rangle^{\otimes n}\right) \\
& -\sum_{g=0}^{\infty} g_{s}{ }^{2 g} \sum_{p, m, n=0}^{\infty} \frac{1}{p ! m ! n !} \int_{\mathcal{R}_{g, m+1, n}} \Omega_{6 g-5+2 m+2 n+2}^{(g, m+1, n)}\left[\widehat{U}_{g, m+1, n}\right]\left(\mathcal{G}\left[(\mathcal{G} \Phi) \Psi^{p}\right]_{N S},\left|\Psi_{N S}\right\rangle^{\otimes m},\left|\Psi_{R}\right\rangle^{\otimes n}\right) \\
& -\sum_{g=0}^{\infty} g_{s}{ }^{2 g} \sum_{p, m, n=0}^{\infty} \frac{1}{p ! m ! n !} \int_{\mathcal{R}_{g, m, n+1}} \Omega_{6 g-5+2 m+2 n+2}^{(g, m, n+1)}\left[\widehat{U}_{g, m, n+1}\right]\left(\left|\Psi_{N S}\right\rangle^{\otimes m}, \mathcal{G}\left[(\mathcal{G} \Phi) \Psi^{p}\right]_{R},\left|\Psi_{R}\right\rangle^{\otimes n}\right) .
\end{aligned}
$$

The terms involving $\left|\Phi_{N S}\right\rangle$ in the above expression are given by

$$
\begin{aligned}
& \left\langle\Phi_{N S}\left|c_{0}^{-}\right| \tilde{\delta} \mathcal{E}\right\rangle \\
& =-\sum_{g=0}^{\infty} g_{s}{ }^{2 g} \sum_{m, n=0}^{\infty} \frac{1}{m ! n !} \int_{\mathcal{R}_{g, m+1, n}} \Omega_{6 g-5+2 m+2 n+2}^{(g, m+1, n)}\left[\widehat{U}_{g, m+1, n}\right]\left(Q_{B} \mathcal{G}\left|\Phi_{N S}\right\rangle,\left|\Psi_{N S}\right\rangle^{\otimes m},\left|\Psi_{R}\right\rangle^{\otimes n}\right) \\
& -\sum_{g=0}^{\infty} g_{s}{ }^{2 g} \sum_{p, m, n=0}^{\infty} \frac{1}{p ! m ! n !} \int_{\mathcal{R}_{g, m+1, n}} \Omega_{6 g-5+2 m+2 n+2}^{(g, m+1, n)}\left[\widehat{U}_{g, m+1, n}\right]\left(\mathcal{G}\left[\left(\mathcal{G} \Phi_{N S}\right) \Psi^{p}\right]_{N S},\left|\Psi_{N S}\right\rangle^{\otimes m},\left|\Psi_{R}\right\rangle^{\otimes n}\right) \\
& -\sum_{g=0}^{\infty} g_{s}{ }^{2 g} \sum_{p, m, n=0}^{\infty} \frac{1}{p ! m ! n !} \int_{\mathcal{R}_{g, m, n+1}} \Omega_{6 g-5+2 m+2 n+2}^{(g, m, n+1)}\left[\widehat{U}_{g, m, n+1}\right]\left(\left|\Psi_{N S}\right\rangle^{\otimes m}, \mathcal{G}\left[\left(\mathcal{G} \Phi_{N S}\right) \Psi^{p}\right]_{R},\left|\Psi_{R}\right\rangle^{\otimes n}\right) \\
& \equiv J_{1}+J_{2}+J_{3}
\end{aligned}
$$

where $J_{1}, J_{2}, J_{3}$ are the three terms on the right hand side of this expression. Our goal is to show that (5.8) and (5.12) are equal when $|\Psi\rangle$ satisfies its equation of motion. First we note that

$$
I_{1}-J_{1}=0
$$

Next we use (3.8) and (5.8) to write ${ }^{9}$

$$
\begin{aligned}
I_{4}= & -\sum_{g_{1}, g_{2}} g_{s}{ }^{2\left(g_{1}+g_{2}\right)} \sum_{m_{1}, m_{2}, n_{1}, n_{2} \geq 0} \frac{1}{m_{1} ! m_{2} ! n_{1} ! n_{2} !} \\
& \times \int_{\mathcal{R}_{g_{1}, m_{1}+2, n_{1}}} \Omega_{6 g_{1}-5+2\left(m_{1}+n_{1}+2\right)}^{\left(g_{1}, m_{1}+2, n_{1}\right)}\left[\widehat{U}_{g_{1}, m_{1}+2, n_{1}}\right]\left(\mathcal{G}\left|\Phi_{N S}\right\rangle,\left|\Psi_{N S}\right\rangle^{\otimes m_{1}},\left|\tilde{\varphi}_{r}\right\rangle,\left|\Psi_{R}\right\rangle^{\otimes n_{1}}\right) \\
& \times \int_{\mathcal{R}_{g_{2}, m_{2}+1, n_{2}}} \Omega_{6 g_{2}-6+2\left(m_{2}+n_{2}+1\right)}^{\left(g_{2}, m_{2}+1, n_{2}\right)}\left(\mathcal{G}\left|\tilde{\varphi}^{r}\right\rangle,\left|\Psi_{N S}\right\rangle^{\otimes m_{2}},\left|\Psi_{R}\right\rangle^{\otimes n_{2}}\right)
\end{aligned}
$$
$\widehat{U}_{g, m, n}{ }^{\mathrm{As}}$.

${ }^{9}$ As in [1], we need slight generalization of (3.8) to take into account the contraction of $\Omega_{p}^{(g, m, n)}$ with 


$$
\begin{aligned}
& -\sum_{g_{1}, g_{2}} g_{s}{ }^{2\left(g_{1}+g_{2}\right)} \sum_{m_{1}, m_{2}, n_{1}, n_{2} \geq 0} \frac{1}{m_{1} ! m_{2} ! n_{1} ! n_{2} !} \\
& \times \int_{\mathcal{R}_{g_{1}, m_{1}+2, n_{1}}} \Omega_{6 g_{1}-6+2\left(m_{1}+n_{1}+2\right)}^{\left(g_{1}, m_{1}+2, n_{1}\right)}\left(\mathcal{G}\left|\Phi_{N S}\right\rangle,\left|\Psi_{N S}\right\rangle^{\otimes m_{1}},\left|\tilde{\varphi}_{r}\right\rangle,\left|\Psi_{R}\right\rangle^{\otimes n_{1}}\right) \\
& \times \int_{\mathcal{R}_{g_{2}, m_{2}+1, n_{2}}} \Omega_{6 g_{2}-5+2\left(m_{2}+n_{2}+1\right)}^{\left(g_{2}, m_{2}+1, n_{2}\right)}\left[\widehat{U}_{g_{2}, m_{2}+1, n_{2}}\right]\left(\mathcal{G}\left|\tilde{\varphi}^{r}\right\rangle,\left|\Psi_{N S}\right\rangle^{\otimes m_{2}},\left|\Psi_{R}\right\rangle^{\otimes n_{2}}\right)
\end{aligned}
$$

where we have used the fact that we get an extra minus sign from the interchange of the operation of integrating over the angular variable $\theta$ of the plumbing fixture relation and contraction with a vector field $\widehat{U}$. This cancels a minus sign coming from the $\prod_{i} \tilde{\sigma}_{i}$ factors in (3.8). Note also that to maintain uniformity with the corresponding analysis involving terms with Ramond degeneration, we have inserted a factor of $\mathcal{G}$ in front of the basis states $\left|\tilde{\varphi}^{r}\right\rangle$ of $\mathcal{H}_{N S}$ even though acting on NS sector states $\mathcal{G}$ reduces to the identity operator. Using (4.2) and (4.6), eq.(5.14) can be rewritten as

$$
\begin{aligned}
& I_{4}=-\sum_{g_{1}} g_{s}{ }^{2 g_{1}} \sum_{m_{1}, m_{2}, n_{1}, n_{2} \geq 0} \frac{1}{m_{1} ! m_{2} ! n_{1} ! n_{2} !} \\
& \times \int_{\mathcal{R}_{g_{1}, m_{1}+2, n_{1}}}^{\Omega_{6 g_{1}-5+2\left(m_{1}+n_{1}+2\right)}^{\left(g_{1}, m_{1}+2, n_{1}\right)}}\left[\widehat{U}_{g_{1}, m_{1}+2, n_{1}}\right]\left(\mathcal{G}\left|\Phi_{N S}\right\rangle,\left|\Psi_{N S}\right\rangle^{\otimes m_{1}}, \mathcal{G}\left[\left(\Psi_{N S}\right)^{m_{2}}\left(\Psi_{R}\right)^{n_{2}}\right]_{N S},\left|\Psi_{R}\right\rangle^{\otimes n_{1}}\right) \\
& -\sum_{g_{2}} g_{s}{ }^{2 g_{2}} \sum_{m_{1}, m_{2}, n_{1}, n_{2} \geq 0} \frac{1}{m_{1} ! m_{2} ! n_{1} ! n_{2} !} \\
& \times \int_{\mathcal{R}_{g_{2}, m_{2}+1, n_{2}}}^{\Omega_{6 g_{2}-5+2\left(m_{2}+n_{2}+1\right)}^{\left(g_{2}, m_{2}+1, n_{2}\right)}\left[\widehat{U}_{g_{2}, m_{2}+1, n_{2}}\right]\left(\mathcal{G}\left[\left(\mathcal{G} \Phi_{N S}\right)\left(\Psi_{N S}\right)^{m_{1}}\left(\Psi_{R}\right)^{n_{1}}\right]_{N S},\left|\Psi_{N S}\right\rangle^{\otimes m_{2}},\left|\Psi_{R}\right\rangle^{\otimes n_{2}}\right) .}
\end{aligned}
$$

We now see that the first term on the right hand side of (5.15), when added to $I_{2}$ defined in (5.8), vanishes after using equations of motion. On the other hand the second term on the right hand side of $(5.15)$ is equal to $J_{2}$ defined in (5.12). Thus we have

$$
I_{2}+I_{4}-J_{2}=0
$$

In exactly the same way one can show that

$$
I_{3}+I_{5}-J_{3}=0 .
$$

Combining (5.13), (5.16) and (5.17) we see that upon using equation of motion we have

$$
\left(I_{1}+I_{2}+I_{3}+I_{4}+I_{5}\right)-\left(J_{1}+J_{2}+J_{3}\right)=0 .
$$

A similar analysis can be carried out involving the terms involving $\left|\Phi_{R}\right\rangle$. This establishes (5.1).

Using the results above one can now show in a straightforward manner that the renormalized mass is independent of the choice of the local coordinates and PCO locations used to define the off-shell amplitudes and the 1PI effective theory. For this we use the fact that to determine the renormalized physical masses we need to examine the zero eigenvalues of the gauge invariant kinetic operator giving the linearized equations of motion around the classical solution $\left|\Psi_{c l}\right\rangle$ representing the vacuum. The zero eigenvalues which exist for 
all values of the momentum vector $k$ correspond to pure gauge states. On the other hand the zero eigenvalues which exist only for special values of $k^{2}$ correspond to physical states and the values of $-k^{2}$ at which the zero eigenvalues appear give the physical renormalized mass $^{2}$. As discussed in detail in [1], the fact that a change in local coordinates and PCO locations correspond to a field redefinition means that these transformations do not affect the (non-)existence of the zero eigenvalue at a given momentum, - they only change the form of the corresponding eigenstate. Thus the values of $-k^{2}$ at which the zero eigenvalues appear remain unchanged, showing that the physical renormalized mass ${ }^{2}$ are not affected by the change in the choice of local coordinates and/or PCO locations.

\section{Space-time supersymmetry}

In heterotic string theory the gauge transformation parameter $|\Lambda\rangle$ appearing in (4.16) contains an R sector component $\left|\Lambda_{R}\right\rangle$ which is a state of ghost number 1 and picture number $-1 / 2$. This includes local supersymmetry transformations, just as $\left|\Lambda_{N S}\right\rangle$ includes general coordinate and local gauge transformations. In type II theories the local supersymmetry transformations are contained in $\left|\Lambda_{R N S}\right\rangle$ and $\left|\Lambda_{N S R}\right\rangle$.

Our interest here is in understanding global supersymmetry transformations. These are special choices of $|\Lambda\rangle$ which leave the vacuum invariant. Our goal will be to develop a systematic procedure for finding such $|\Lambda\rangle$ 's. For definiteness we focus on the heterotic string theory - generalization to type II string theories is straightforward. We begin by recalling that in general the vacuum is not given by the $|\Psi\rangle=0$ configuration instead it corresponds to some specific configuration $\left|\Psi_{c l}\right\rangle$ whose systematic construction was described in [1]. Thus the global supersymmetry transformation parameter $\left|\Lambda_{R}\right\rangle$, which by definition will be taken to carry zero momentum, must satisfy

$$
Q_{B}\left|\Lambda_{R}\right\rangle+\sum_{n=0}^{\infty} \frac{1}{n !} \mathcal{X}_{0}\left[\Psi_{c l}^{n} \Lambda_{R}\right]=0 .
$$

We can look for a solution in a power series in the string coupling $g_{s}$ by beginning with the leading order solution

$$
\left|\Lambda_{0}\right\rangle=\left|c e^{-\phi / 2} S_{\alpha}\right\rangle,
$$

where $S_{\alpha}$ is an appropriate dimension $(0,5 / 8)$ spin field from the matter sector. We then compute the corrections iteratively by solving ${ }^{10}$

$$
Q_{B}\left|\Lambda_{k}\right\rangle=-\sum_{n=0}^{\infty} \frac{1}{n !} \mathcal{X}_{0}\left[\Psi_{c l}^{n} \Lambda_{k-1}\right]+\mathcal{O}\left(g_{s}{ }^{k+1}\right) .
$$

Here $\left|\Lambda_{k}\right\rangle$ is the approximation to the global supersymmetry transformation parameter $\left|\Lambda_{R}\right\rangle$ to order $g_{s}{ }^{k}$. For consistency we need to ensure that if $\left|\Lambda_{k-1}\right\rangle$ satisfies the above equation with $k$ replaced by $(k-1)$, then we must have

$$
Q_{B} \sum_{n=0}^{\infty} \frac{1}{n !} \mathcal{X}_{0}\left[\Psi_{c l}^{n} \Lambda_{k-1}\right]=\mathcal{O}\left(g_{s}{ }^{k+1}\right) .
$$

\footnotetext{
${ }^{10}$ Even though the usual perturbation expansion in string theory is in powers of $g_{s}{ }^{2}$, we take the expansion to be in powers of $g_{s}$ since in some cases $\left|\Psi_{c l}\right\rangle$ may be of order $g_{s}[1,44]$.
} 
The proof of this involves straightforward manipulation using (4.8) and the equations of motion (4.15) for $\left|\Psi_{c l}\right\rangle$ and was given in [1] in a slightly different context while discussing mass renormalization. An obstruction arises if the right hand side of (6.3), which is a state of ghost number 2 and picture number $-1 / 2$, contains a non-trivial element of the BRST cohomology; this is allowed by (6.4). A basis of such states is provided by the zero momentum states of physical massless fermions. If the right hand side of (6.3) has non-zero component along such a state then supersymmetry is broken and the corresponding state represents the zero momentum goldstino state associated with the broken supersymmetry. Thus the condition for unbroken supersymmetry will require that the right hand side of (6.3) does not have any component along these possible goldstino states. Since the BPZ inner product with $c_{0}^{-}$insertion pairs states of ghost number 2 and picture number $-1 / 2$ with states of ghost number 3 and picture number $-3 / 2$, the above condition can also be expressed as

$$
\left\langle\phi\left|c_{0}^{-} \sum_{n=0}^{\infty} \frac{1}{n !} \mathcal{X}_{0}\right|\left[\Psi_{c l}^{n} \Lambda_{k-1}\right]\right\rangle=\mathcal{O}\left(g_{s}{ }^{k+1}\right),
$$

for any BRST invariant state $|\phi\rangle$ of ghost number 3 and picture number $-3 / 2$. The possible non-trivial constraints come from states with $L_{0}^{+}$eigenvalue 0 , since BRST invariant states with $L_{0}^{+} \neq 0$ are also BRST trivial.

We can make connection with the criteria given in $[15,16]$ by noting that if such obstructions are absent up to a given order then to that order we can solve (6.3) by taking

$$
\left|\Lambda_{k}\right\rangle=-\sum_{n=0}^{\infty} \frac{1}{n !} \frac{b_{0}^{+}}{L_{0}^{+}}(1-\mathbf{P}) \mathcal{X}_{0}\left[\Psi_{c l}^{n} \Lambda_{k-1}\right]+\left|\tau_{k}\right\rangle,
$$

where $\mathbf{P}$ denotes the projection operator into $L_{0}^{+}=0$ states and $\left|\tau_{k}\right\rangle$ is an $L_{0}^{+}=0$ state satisfying

$$
Q_{B}\left|\tau_{k}\right\rangle=-\sum_{n=0}^{\infty} \frac{1}{n !} \mathbf{P} \mathcal{X}_{0}\left[\Psi_{c l}^{n} \Lambda_{k-1}\right]
$$

In order to continue this construction to the next order we need that (6.5) should hold with $k$ replaced by $k+1$. If we replace $\mathbf{P}$ by 0 (i.e. ignore the special treatment of the $L_{0}^{+}=0$ states) and make a similar operation in the construction of $\left|\Psi_{c l}\right\rangle$ described in [1], then this condition can be shown to be equivalent to the requirement that the full two point function of $|\phi\rangle$ and $\left|\Lambda_{0}\right\rangle$ - including 1PI and 1PR contributions - vanish to order $g_{s}{ }^{k+1}$. This is precisely the condition for unbroken supersymmetry described in $[15,16]$. However this two point function has to be regularized to deal with divergences associated with separating type degenerations of Riemann surfaces at the intermediate stage of the computation. In contrast our construction removes these divergences from the beginning by inserting the projection operator $(1-\mathbf{P})$ in (6.6) and compensates for this by inclusion of the additional state $\left|\tau_{k}\right\rangle$ (and similar operation in the construction of $\left|\Psi_{c l}\right\rangle$ described in [1].) 


\section{Discussion}

We conclude the paper by discussing possible applications of the formalism developed here beyond studying the problems of mass renormalization and vacuum shift.

1. The requirement of gauge invariance puts strong constraint on the 1PI effective theory. Indeed the identity (3.7) which is crucial for proving the infinite dimensional gauge invariance of the 1PI effective theory is also responsible for the fact that the different subspaces of $\widetilde{\mathcal{P}}_{g, m, n}$ associated with different Feynman diagrams of this theory fit together to give the full integration cycle. Given this, one might wonder if the infinite dimensional gauge symmetry could also be useful for constraining the non-perturbative corrections to the 1PI effective theory. It is worth examining this question further since the current approach to the study of non-perturbative effects in string theory is based mostly on the intuitions from the low energy theory.

2. Formulating string theory in the RR background has been an open problem. The 1PI effective theory could provide a way out for weak RR background, since we could construct the 1PI theory in a background where there is no RR field, and then study the effect of switching on RR background by expanding the original 1PI equations of motion around the new background in powers of the RR background field. This could for example give a way to study $g_{s}$ and $1 / L$ corrections systematically in string theory on AdS spaces of size $L$, since typically in the large $L$ limit the RR field strength is small (locally).

\section{Acknowledgments}

We thank Roji Pius, Arnab Rudra, Edward Witten and Barton Zwiebach for useful discussions and Barton Zwiebach for his valuable comments on an earlier version of the manuscript. This work was supported in part by the DAE project 12-R\&D-HRI-5.02-0303 and J. C. Bose fellowship of the Department of Science and Technology, India.

Open Access. This article is distributed under the terms of the Creative Commons Attribution License (CC-BY 4.0), which permits any use, distribution and reproduction in any medium, provided the original author(s) and source are credited.

\section{References}

[1] A. Sen, Gauge Invariant 1PI Effective Action for Superstring Field Theory, JHEP 06 (2015) 022 [arXiv: 1411.7478] [INSPIRE].

[2] D. Friedan, E.J. Martinec and S.H. Shenker, Conformal Invariance, Supersymmetry and String Theory, Nucl. Phys. B 271 (1986) 93 [inSPIRE].

[3] E. Witten, Interacting Field Theory of Open Superstrings, Nucl. Phys. B 276 (1986) 291 [INSPIRE].

[4] N. Berkovits, SuperPoincaré invariant superstring field theory, Nucl. Phys. B 450 (1995) 90 [Erratum ibid. B 459 (1996) 439] [hep-th/9503099] [INSPIRE]. 
[5] N. Berkovits, The Ramond sector of open superstring field theory, JHEP 11 (2001) 047 [hep-th/0109100] [INSPIRE].

[6] Y. Okawa and B. Zwiebach, Heterotic string field theory, JHEP 07 (2004) 042 [hep-th/0406212] [INSPIRE].

[7] N. Berkovits, Y. Okawa and B. Zwiebach, WZW-like action for heterotic string field theory, JHEP 11 (2004) 038 [hep-th/0409018] [INSPIRE].

[8] H. Kunitomo, The Ramond Sector of Heterotic String Field Theory, PTEP 2014 (2014) 043B01 [arXiv: 1312.7197] [INSPIRE].

[9] H. Kunitomo, Symmetries and Feynman rules for the Ramond sector in open superstring field theory, PTEP 2015 (2015) 033B11 [arXiv:1412.5281] [INSPIRE].

[10] A. Sen, Off-shell Amplitudes in Superstring Theory, Fortsch. Phys. 63 (2015) 149 [arXiv: 1408.0571] [INSPIRE].

[11] N. Berkovits and B. Zwiebach, On the picture dependence of Ramond-Ramond cohomology, Nucl. Phys. B 523 (1998) 311 [hep-th/9711087] [INSPIRE].

[12] T. Erler, S. Konopka and I. Sachs, NS-NS Sector of Closed Superstring Field Theory, JHEP 08 (2014) 158 [arXiv: 1403.0940] [INSPIRE].

[13] R. Pius, A. Rudra and A. Sen, Mass Renormalization in String Theory: Special States, JHEP 07 (2014) 058 [arXiv: 1311.1257] [INSPIRE].

[14] R. Pius, A. Rudra and A. Sen, Mass Renormalization in String Theory: General States, JHEP 07 (2014) 062 [arXiv:1401.7014] [INSPIRE].

[15] E. Witten, Superstring Perturbation Theory Revisited, arXiv:1209.5461 [INSPIRE].

[16] E. Witten, More On Superstring Perturbation Theory, arXiv:1304.2832 [INSPIRE].

[17] A. Belopolsky, De Rham cohomology of the supermanifolds and superstring BRST cohomology, Phys. Lett. B 403 (1997) 47 [hep-th/9609220] [INSPIRE].

[18] A. Belopolsky, New geometrical approach to superstrings, hep-th/9703183 [INSPIRE].

[19] A. Belopolsky, Picture changing operators in supergeometry and superstring theory, hep-th/9706033 [INSPIRE].

[20] E. D'Hoker and D.H. Phong, Two loop superstrings. 1. Main formulas, Phys. Lett. B 529 (2002) 241 [hep-th/0110247] [INSPIRE].

[21] E. D'Hoker and D.H. Phong, Two loop superstrings. 2. The chiral measure on moduli space, Nucl. Phys. B 636 (2002) 3 [hep-th/0110283] [INSPIRE].

[22] E. D'Hoker and D.H. Phong, Two loop superstrings. 3. Slice independence and absence of ambiguities, Nucl. Phys. B 636 (2002) 61 [hep-th/0111016] [INSPIRE].

[23] E. D'Hoker and D.H. Phong, Two loop superstrings. 4. The cosmological constant and modular forms, Nucl. Phys. B 639 (2002) 129 [hep-th/0111040] [INSPIRE].

[24] E. D'Hoker and D.H. Phong, Two-loop superstrings. 5. Gauge slice independence of the N-point function, Nucl. Phys. B 715 (2005) 91 [hep-th/0501196] [INSPIRE].

[25] E. D'Hoker and D.H. Phong, Two-loop superstrings. 6. Non-renormalization theorems and the 4-point function, Nucl. Phys. B 715 (2005) 3 [hep-th/0501197] [INSPIRE]. 
[26] E. D'Hoker and D.H. Phong, Two-Loop Superstrings. 7. Cohomology of Chiral Amplitudes, Nucl. Phys. B 804 (2008) 421 [arXiv:0711.4314] [INSPIRE].

[27] E. Witten, Notes On Supermanifolds and Integration, arXiv:1209.2199 [INSPIRE].

[28] E. Witten, Notes On Super Riemann Surfaces And Their Moduli, arXiv:1209.2459 [INSPIRE].

[29] E. Witten, Notes On Holomorphic String And Superstring Theory Measures Of Low Genus, arXiv:1306.3621 [INSPIRE].

[30] R. Donagi and E. Witten, Supermoduli Space Is Not Projected, arXiv:1304.7798 [INSPIRE].

[31] R. Donagi and E. Witten, Super Atiyah classes and obstructions to splitting of supermoduli space, arXiv: 1404.6257 [INSPIRE].

[32] E. D'Hoker and D.H. Phong, Two-loop vacuum energy for Calabi-Yau orbifold models, Nucl. Phys. B 877 (2013) 343 [arXiv: 1307.1749] [InSPIRE].

[33] E. D'Hoker, Topics in Two-Loop Superstring Perturbation Theory, arXiv:1403.5494 [INSPIRE].

[34] T. Erler, S. Konopka and I. Sachs, Resolving Witten's superstring field theory, JHEP 04 (2014) 150 [arXiv: 1312.2948] [INSPIRE].

[35] H. Matsunaga, Nonlinear gauge invariance and WZW-like action for NS-NS superstring field theory, arXiv: 1407.8485 [INSPIRE].

[36] E.P. Verlinde and H.L. Verlinde, Multiloop Calculations in Covariant Superstring Theory, Phys. Lett. B 192 (1987) 95 [inSPIRE].

[37] P.C. Nelson, Covariant Insertion of General Vertex Operators, Phys. Rev. Lett. 62 (1989) 993 [INSPIRE].

[38] B. Zwiebach, Closed string field theory: Quantum action and the B-V master equation, Nucl. Phys. B 390 (1993) 33 [hep-th/9206084] [INSPIRE].

[39] O. Lechtenfeld, Superconformal ghost correlations on Riemann surfaces, Phys. Lett. B 232 (1989) 193 [inSPIRE].

[40] A. Morozov, Straightforward proof of Lechtenfeld's formula for $\beta, \gamma$-correlator, Phys. Lett. B 234 (1990) 15 [Yad. Fiz. 51 (1990) 301] [Sov. J. Nucl. Phys. 51 (1990) 190] [INSPIRE].

[41] A. Berera, Unitary string amplitudes, Nucl. Phys. B 411 (1994) 157 [INSPIRE].

[42] E. Witten, The Feynman i€ in String Theory, JHEP 04 (2015) 055 [arXiv:1307.5124] [INSPIRE].

[43] H. Hata and B. Zwiebach, Developing the covariant Batalin-Vilkovisky approach to string theory, Annals Phys. 229 (1994) 177 [hep-th/9301097] [INSPIRE].

[44] R. Pius, A. Rudra and A. Sen, String Perturbation Theory Around Dynamically Shifted Vacuum, JHEP 10 (2014) 70 [arXiv:1404.6254] [INSPIRE]. 